\title{
المواد النفسية الجديدة (NPS)
}

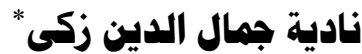

شهدت السنوات الأخيرة توليف المئات من المواد النفسية الجديدة (NPS) التى تم تصنيعها لكى لكى لكي

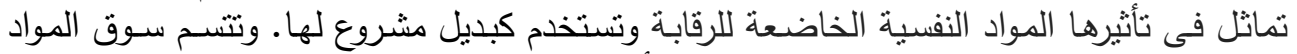

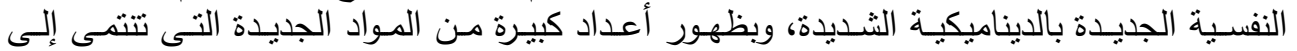

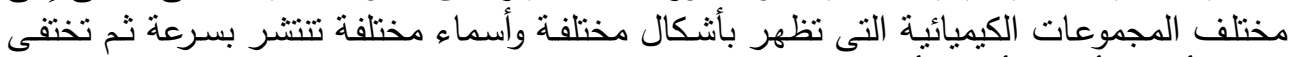

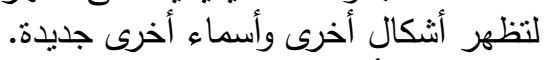

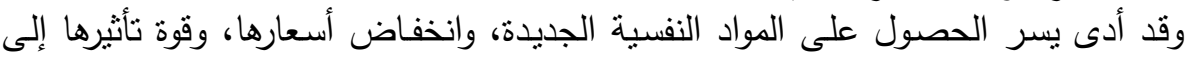

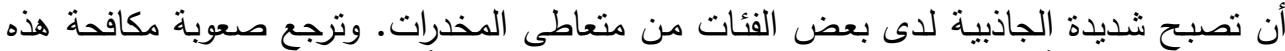

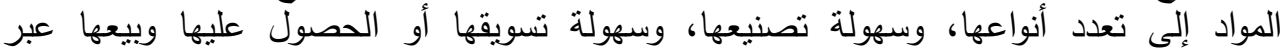

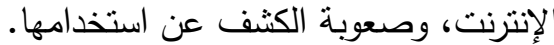

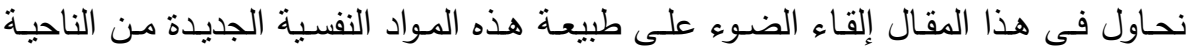

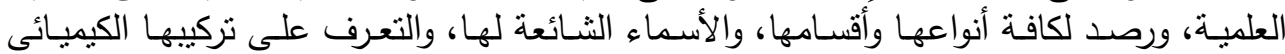

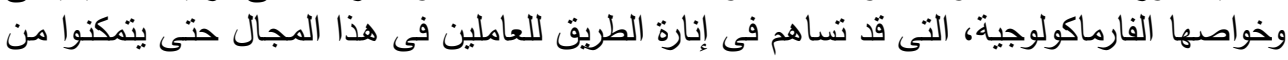

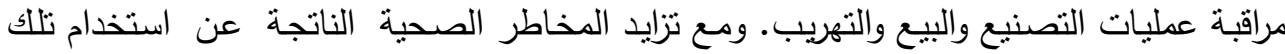

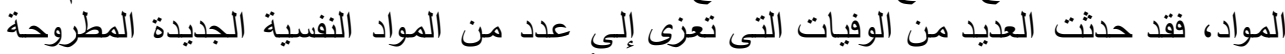

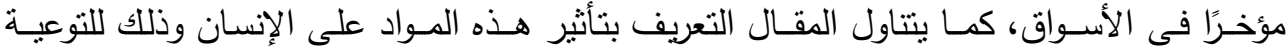

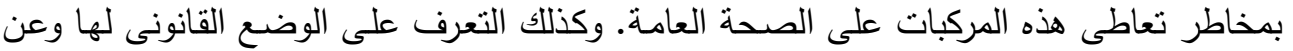

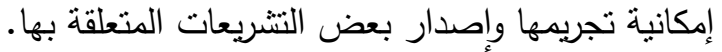

\section{المقدهة}

شهد المجتمع المصرى فى الآونة الأخيرة تحولات اقتصادية واجتماعية وسياسية فارقة، تشكل فى مجملها بيئة مواتية لانتشار وتعاطى المواد المخدرة، وفى الوقت الت التهرة نفسه فإن التغيرات غير المسبوقة التى طرأت على ظاهرة المخدرات على الصعيد العالمى انعكست بطبيعة الحال على السوق المصرى، الأمر الذى أدى إلى تغيرات * أستاذ السموم والمخدرات، المركز القومى للبحوث الاجتماعية والجنائية.

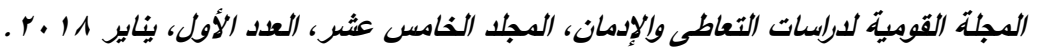


لافتة للنظر فى أسواق المخدِرات الآن، ذلك أن المخدِّرات المتاحة منذ فترة طويلة

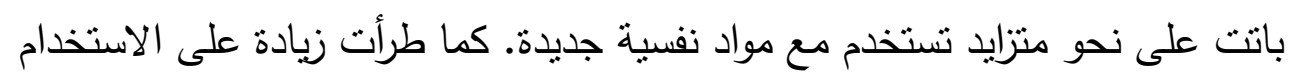

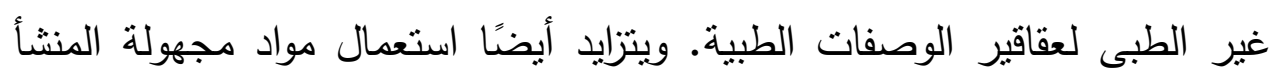
مورَّدة عن طريق قنوات غير مشروعة وتباع كأدوية مزعومة ولكنها معدة للاستعمال غير الطبى، ويلاحَظ أن طائفة المواد والتوليفات المتاحة للمتعاطين باتت أوسع نطاقًا

$$
\text { عن أي وقت مضى. }
$$

فقد انتشر فى الآونة الأخيرة مجموعة كبيرة منتوعة من المخدرات الصناعية

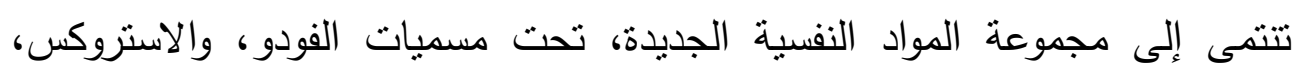

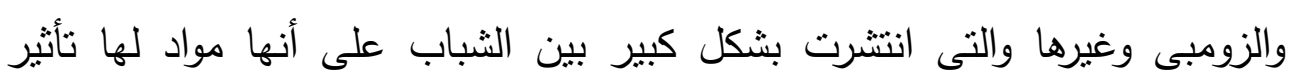

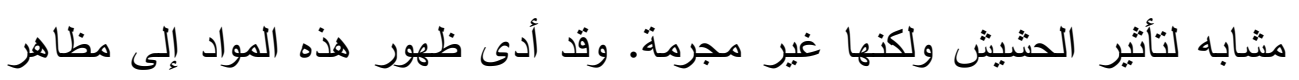

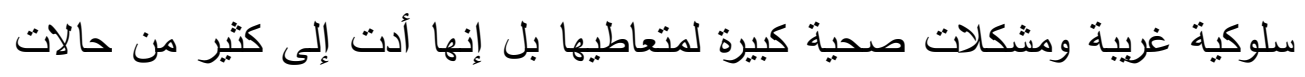
الوفاة.

كما شهدت السنوات الأخيرة توليف المئات من المواد النفسية الجديدة (NPS)

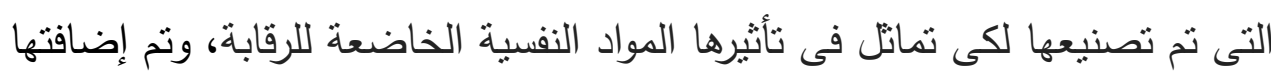

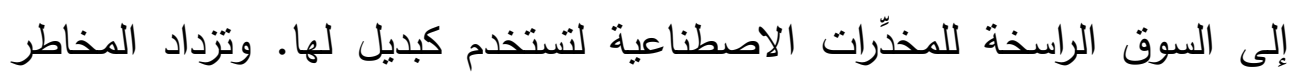

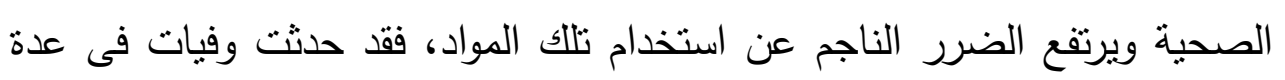

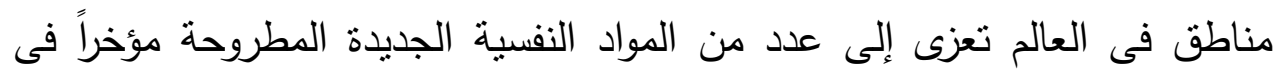
الأسواق والتى لها نفس آثار المواد الأفيونية(1).

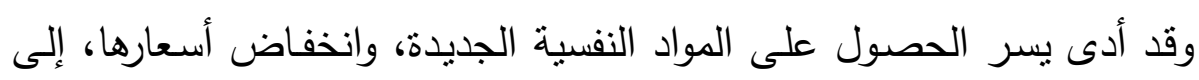

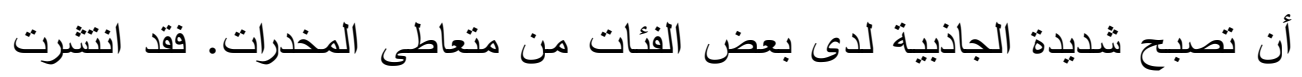

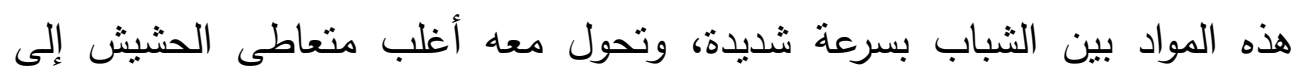

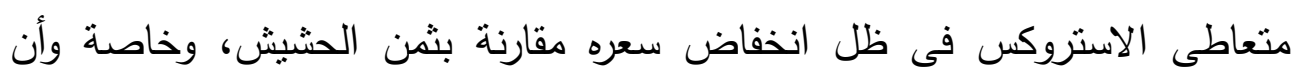


مفعوله أقوى من كل الأنواع المخدرة المنزرعة الأخرى. وترجع صعوبة مكافحة هذه

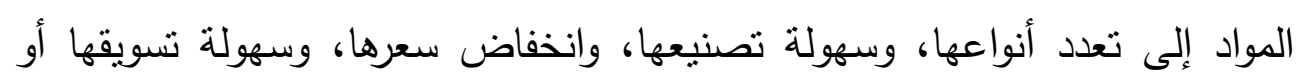

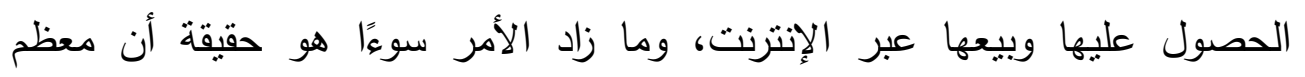

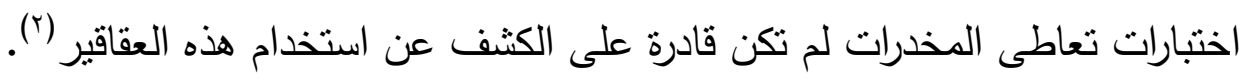

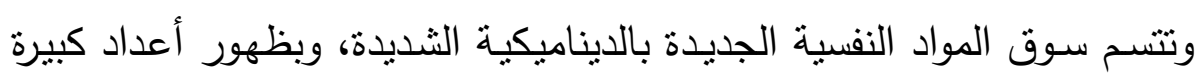

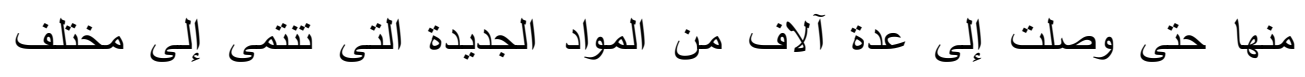

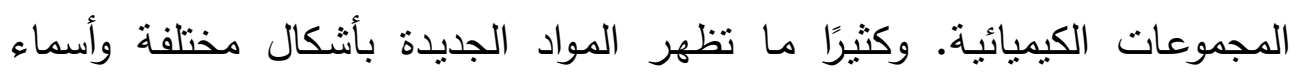
مختلفة، تسوق بسرعة ثم تختفى لتظهر أنشكال أخرى وأسماء أخرى جديدة وهكذا.

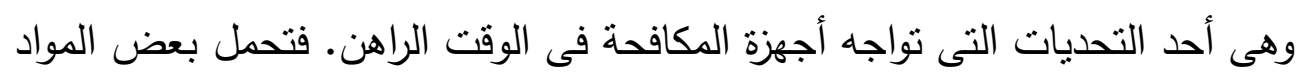

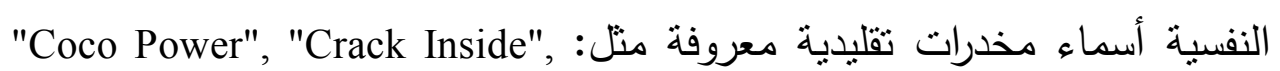
"Snow Blow", "Xtacy", 'Rocket Fuel', "Dynamite", "Euphoria", "Experience Chill", "Herbal Dream", "Synthetic Heroin", "Fake morphine" كما يحمل البعض الآخر أسماء أفلام مشهورة أفلام كرتون منل: "M-ket". "Black mamba", "Clockwork orange" "Mad Alice" and "Pink Panther"), "King أو أسماء حيوانات مثل Scooby snax", "He-Man" and "Kryptonite" أو أسماء أخرى متل: Cobra", "Snow Leopard" and "Dragonfly" "Moon rocks" ففى مصر ظهر مخدر الفودو تلاه مخدر الاستروكس الذى انتشر بشكل كبير

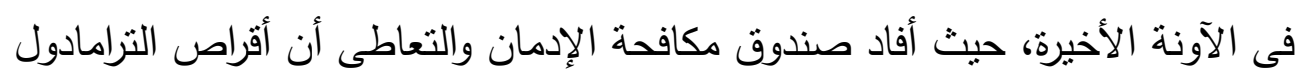

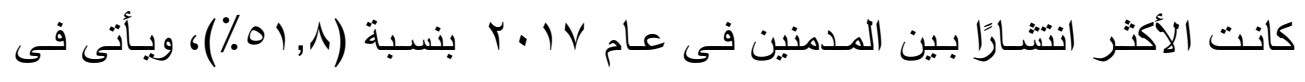

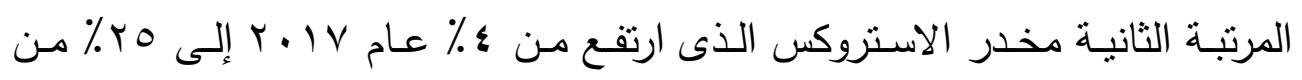

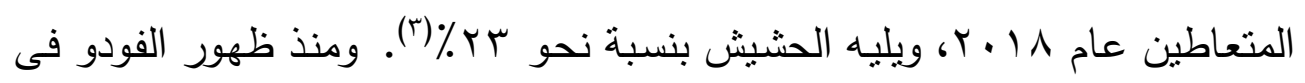
الأسواق المصرية وتلاه مخدر الاستروكس ثنار جدل واسع بشأن هذه المواد الأمر

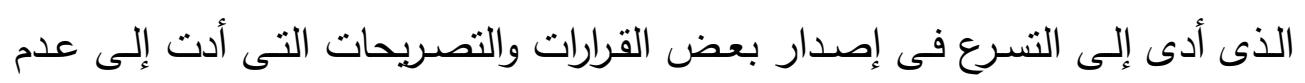


وضوح الرويـة فيمـا يتعلق بمكونـات هذه المخدرات وعن إمكانيـة تجريمها وإصـدار بعض التشريعات المتعلقة بها. وعليه نحاول فى هذا المقال إلقاء الضوء على طبيعة هذه المواد من الناحية العلمية والتى قد تساهم فى إنارة الطريق للعاملين فى هذا المجال.

\section{تعريف المواد النفسية الجديدة}

المواد النفسية الجديدة (NPS) هى مجموعة كبيرة من المواد المختلفة تمائًا فى تركيبها

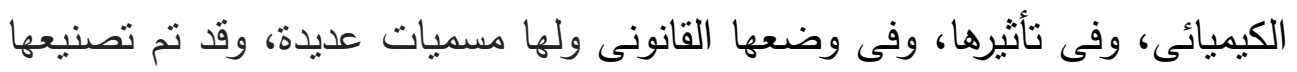

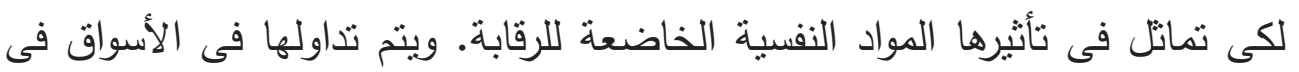
أثكال مختلفة وبالثالى يتم تعاطيها إما عن طريق الفم أو التنخين أو الثم أو الحقن، وغالبًا ما تباع فى شكل خلبط من المواد (๕).

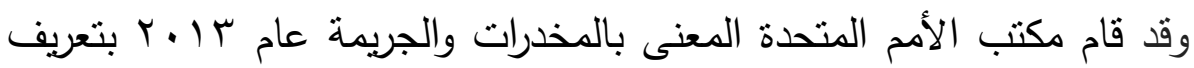

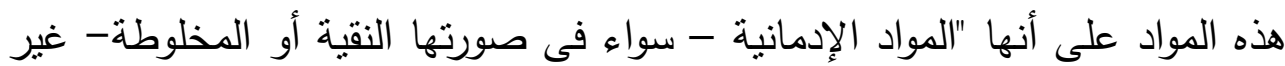

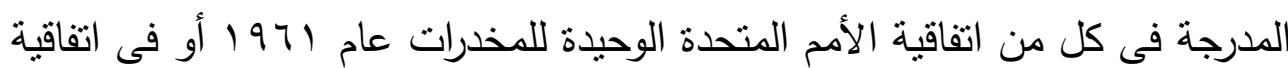

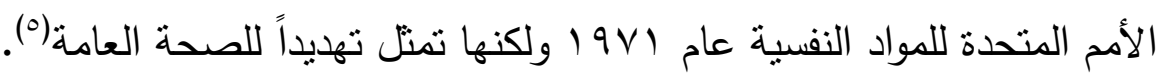

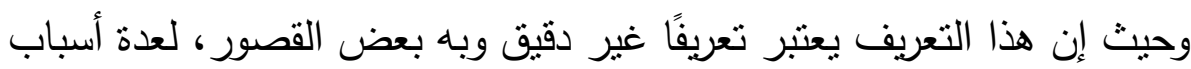

أولاً: لأن هذا التعريف يضم فى طياته مجموعة كبيرة من المواد المختلفة تمامًا

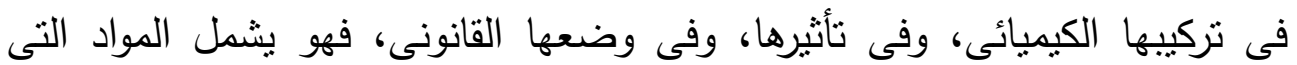

"Emerging Drugs", "Legal Highs", "Research Chemicals", "Designer Drugs", "Club Drug" (Recreational drugs) or "Pharming parties" (Drugs used at certain meetings, parties or private gatherings $)^{(\mathfrak{l})}$. 
ثانيًا: إن هذا التعريف على الرغم من أنه يضم بعض المواد الجديدة بالفعل

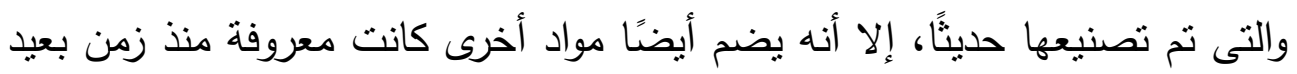

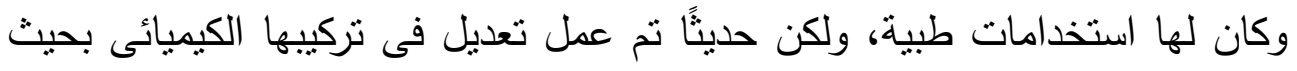

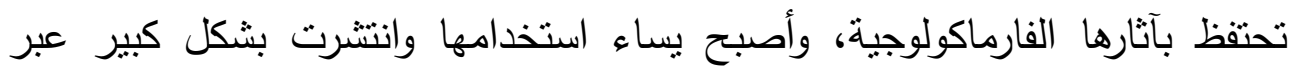

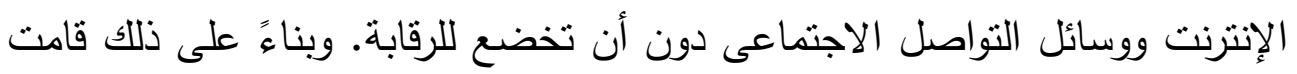

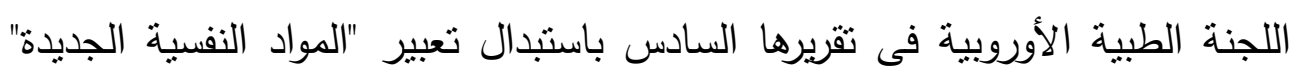
بتعبير آخر هو "Emerging Drugs" وقامت بوضع تعريف لها بأنها: "هى نلألك المواد

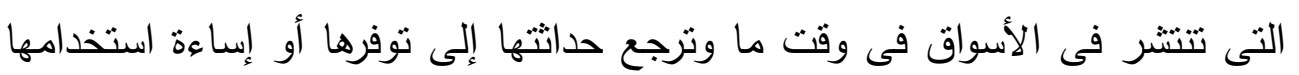
أو تصنيعها وعدم خضوعها للرقابة (V)

\section{الأنهواع المنتلفة للمواد النفسية الجديدة}

المواد النفسية الجديدة (NPS) هى مجموعة كبيرة من المواد مختلفة تمامًا فى تركيبها الكيميائى، وتضم كل من القنبيات المصنعة، والكاثنينون المصنع، والفينيثيل أمين،

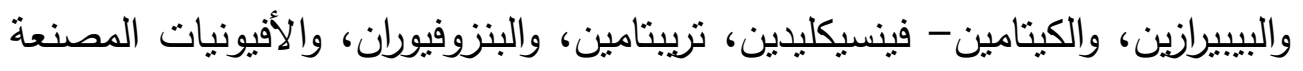

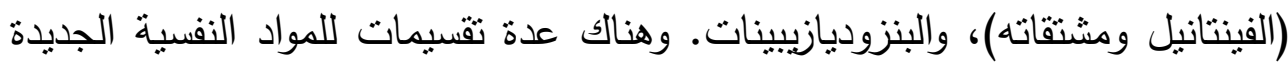

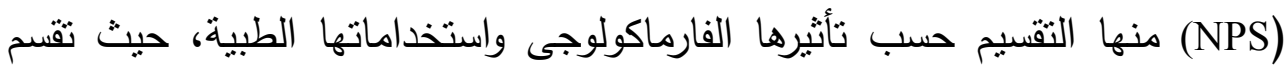

$$
\text { إلى ست مجموعات رئيسية (^) }
$$

1- المنشطات: وهى تماثل فى تأثيرهـا الأمفيتامينات، والكوكايين، والإكستازى،

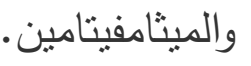

r- القنبيات المصنعة: وهى تماتل فى ثأثنيرها القنبيات. r- المهلوسات: وهى تماتل فى تأثثرها المهلوسات التقليدية مثل عقار الهلوسة الثرات LSD 


$$
\begin{aligned}
& \text { ع - Dissociatives: وهى تماتل فى تأثيرها الكيتامين والفينسيكليدين. } \\
& \text { 0- الأفيونيات المصنعة: وهى تماثل فى تأثثرها المورفين والهيروين. } \\
& \text { צ- المسكنات الدخدرة: وهى أثنباه البنزوديازيبينات. }
\end{aligned}
$$

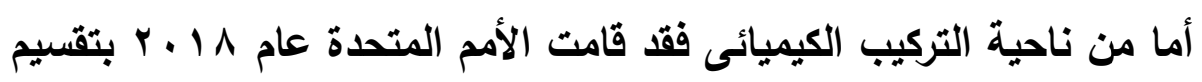

المواد النفسية الجديدة إلى المجموعات التسعة الآتية(9):

أولاً: هجموعة الأهينوإندين Aminoindanes

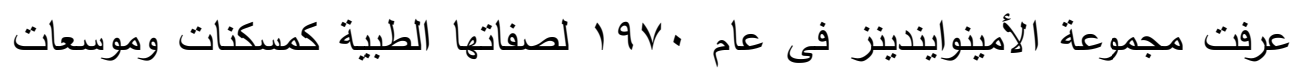

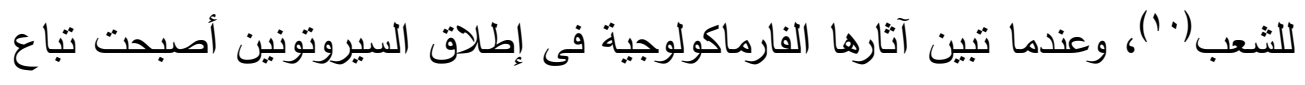

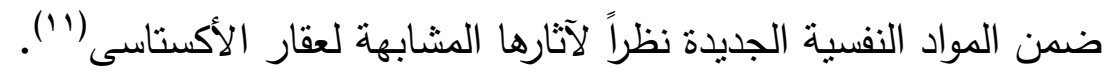

(a)<smiles>CC(N)Cc1ccccc1</smiles>

(b)

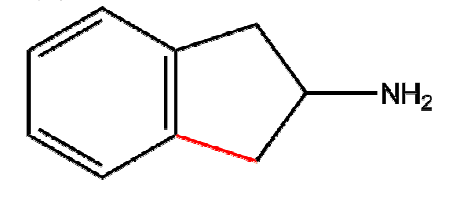

Figure 1: Chemical structures of (a) amphetamine and (b) 2-aminoindane (2-AI).

والعقار النموذج prorotype الذى يشتق منه باقى مركبات هذه المجموعة هو

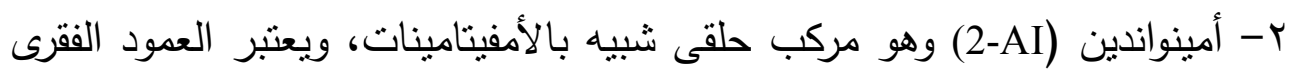

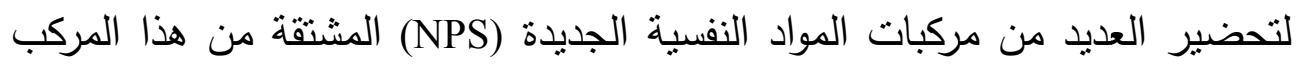
5-IAI (5-iodo-2-aminoindane), MDAI (5,6-methylenedioxy-2-aminoind ( ${ }^{(r)}$ ane) and NM-2AI ( $N$-methyl-2-aminoindane) 
(a)<smiles>NC1Cc2ccccc2C1</smiles>

(c)

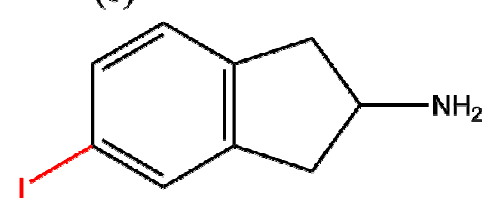

(b)<smiles>NC1Cc2cc3c(cc2C1)OCO3</smiles>

(d)

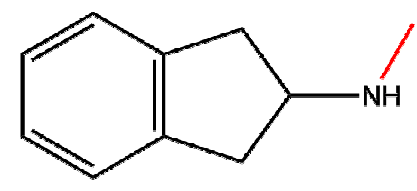

Figure 2: Chemical modifications of the (a) 2-AI backbone are indicated: (b) (MDMAI), (c) (5-IAI) and (d) (NM-2AI).

كانت مركبات الMDAI, 5-IAI and 2-AI هى الأكثز انتشارًا حتى عام 10 • r. ثم تم حديثًا تصنيع مجموعة أخرى من المشثقات منها ال NM-2AI. تعمل هذه المجموعة أساساً كمنشط للجهاز العصبى المركزى، حيث إنها تؤثز فى إنتاج كل من الموصلات العصبية السيروتونين والدوبامين والنورابينيفرين، وهى تمانل فى ذلك تأثير بعض المخدرات التقليدية المعروفة منل الكوكايين والأمفيتامين والميثامفتيامين والإكستاسى. تباع هذه العقاقير تحت أسماء رنانة لجذب المتعاطى فيباع أحد أنواع هذه المجموعة الMDAI فى شكل بودرة تحت مسميات مختلفة منل Party Pills الذهبى)، كما وجد أن MAI يباع مع أقراص الحفلات MDAI)

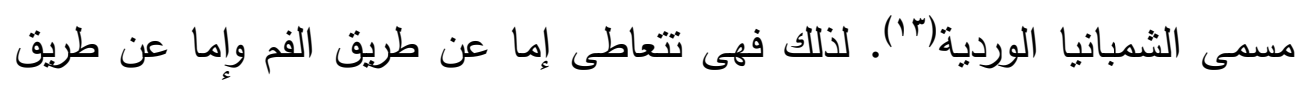
الثم. 


\section{الآثار السلبية لتعاطى هذه المواد}

عقاران من هذه المجموعة وهما الفينفلورامين (بونديمين) والفينفلورامين (ريدوكس) كانا يستخدما فى علاج السمنة فى التسعينيات، ولكن سرعان ما تم سحبهما من الأسواق بعد إصابة مستخدميها بأمراض صمامات القلب(؛).) ولا يوجد دراسات كافية على تأثير هذه المواد على الإنسان.

جميع مشتقات هذه المجموعة غير خاضعة للرقابة الدولية.

ثانيًا: همبموعة شبائه الفينسيكليدين Aryl cycloalkylamines تتدرج هذه المجموعة تحت مركبات الأريل سيكلوأمينات وهى نتبه فى نركيبها الكيميائى كل من عقار الـ ب.س.ب (PCP)، وعقار الكيتامين(10). وقد بدأ تصنيع عقار (PCP) فى عام • 190 وتم تداوله فى الولايات المتحدة الأمريكية كأمبولات بنج، ولكن سرعان ما تم سحبه من الأسواق عند اكتشاف

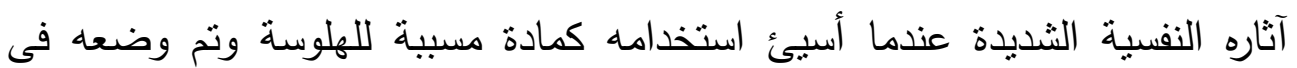
جداول المواد الخاضعة للرقابة الدولية(7). أما الكيتامين فعلى الرغم من استخدامه على نطاق واسع فى المجال الطبى كبنج(v') إلا أنه قد أسئ استخدامه أيضًا مما تسبب فى مشاكل صحية كبيرة، وبالتالى لم يلق استحسانًا كبيرًا لدى المتعاطين. PCP- وقد تم تصنيع عدد من المواد النفسية الجديدة من شبيهات الفينسيكليدين type substances

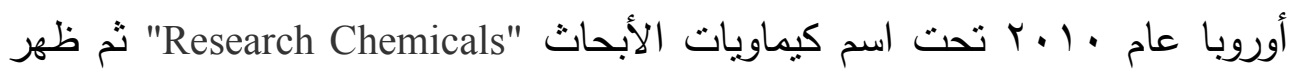

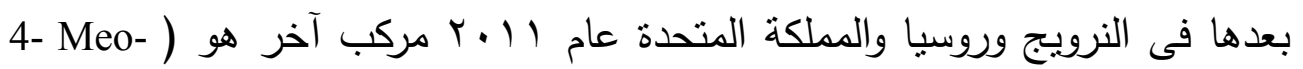
PCP انتشاراً (1^) 
تؤثر مجموعة شبيهات الفينسيكليدين على الجهاز العصبى المركزى وتحدث

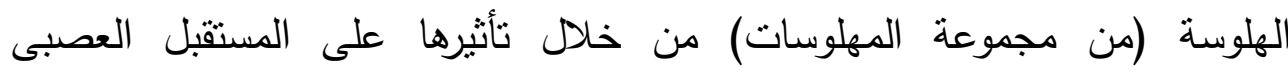
N-Methyl D aspartate receptor (NMDA)

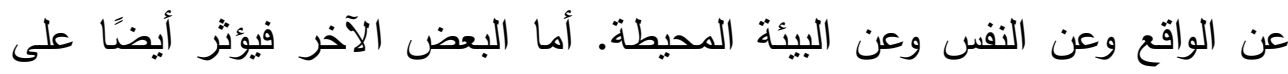

$$
\text { الجهاز العصبى المركزى ويسبب نشاطه (المنشطات). }
$$

\section{الآثار الصحية السلبية}

المعلومات المتاحة عن الآثار السلبية لتعاطى شبائه أو مشتقات الفينسيكليدين محدودة جدًا. ولكن من المعروف أن كل من الفينسيكليدين والكيتامين لهما آثار سمية نفسية وسلوكية كثيرة، كما أن لهما آثار فسيولوجية متعددة. فيتسبب تعاطى الكيتامين فى الى ونى

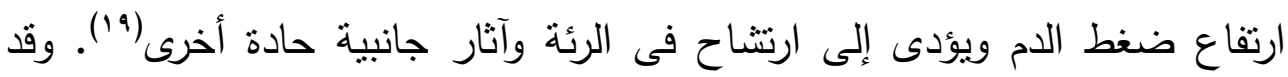

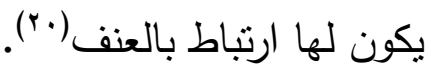

ثالثًا: هبموعة الفينيثيل أهين Phenethyl amines معظم أعضاء هذه المجموعة تتدرج تحت مجموعة المنشطات التى لها آثار نفسية شديدة فهى تعمل كمنشطات للجهاز العصبى المركزى من خلال نأثيرها على إنتاج

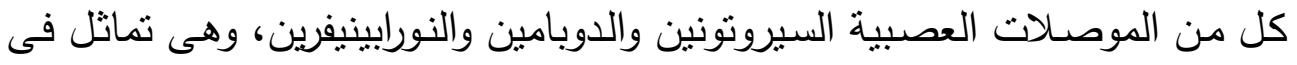

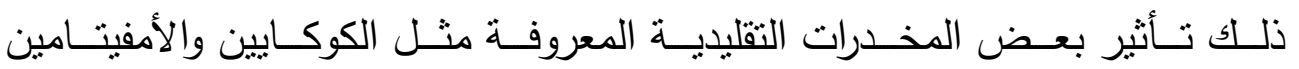
والميثامفتبـامين والمثيلـين دايـوكس ميثامفيتـامين (الإكستاسـى) الخاضـعة للرقابـة

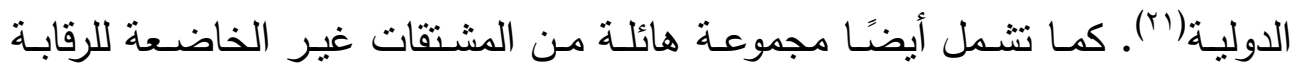

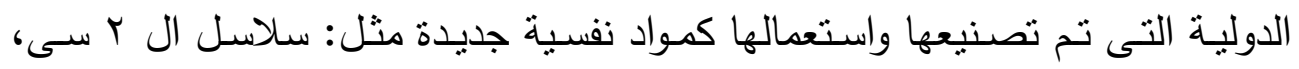
ومشتقات الأمفيتامين سلاسل ال ب دى التى منها (DOI, DOC)، والبنزو داى فيوران 
التى منها (Bromo-Dragonfly, 2C-B-Fly) وغيرها مثل: p-methoxymethamphet amine (PMMA)

وقد وجد أن ال بى إم إم إى PMMA يصنع فى شكل أقراص ويخلط مع عقار آخر من العقاقير الخاضعة للرقابة وهو PMA ويباع فى الأسواق على أنه عقار

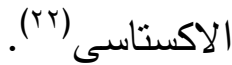

وقد بدأ ظهور هذه المشتقات فى عام 9 . . حين تم ضبط بعض مواد هذه المجموعة فى أمريكا وأوروبا وهى: 2C-E, 2C-I, 4-FA, PMMA، وفى عام 1 . . ب ثم ضبط أنواع أخرى من هذه المجموعة وهى:-4-FMA, 5-APB, 6-APB, and 2C. ومنذ ذلك الحين وأصبحت هذه المواد يتم ضبطها بشكل منزايد. كما أن بعض أعضاء هذه المجموعة يعمل كمهلوسات وهى تماثل فى ذلك مه تأثير المهلوسات التقليدية مثل عقار الهلوسة (LSD) وعقار DMT داى ميثيل تربيتامين.

فى عام 9 \ ا قام العالم شولجين Shulgin بتخليق عدد من المواد المسببة للهلوسة القوية من مادة الميسكالين (وهى من المواد الطبيعية التى تتنمى إلى مجموعة

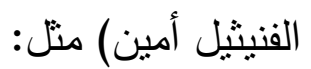
4-bromo-2,5-dimethoxyphenethylamine (2C-B) تصنيع مجموعة كبيرة من مشتقات الميسكالين مثل 2C-B and DOB والتى وجد أن آثارها تفوق بشدة تلك الآتار التى تحدثها المواد المهلوسة الطبيعية(عَ). وتوالت بعد ذللك تصنيع مشتقات مهلوسة أخرى مثل: - Benzodifurans, such as 'FLY' (tetrahy (ro) drobenzodifuranyl) and 'Dragonfly' (benzodifuranyl aminoalkanes) هذه المركبات فى شكل أقراص والبعض الآخر متوفر فى شكل مسـاحيق (بودرة). 'Europa' for 2C-E; '4-FMP', 'para-fluoroamphetamine', 'RDJ' تحت أسماء for 4-FA; and '4-MMA', 'Methyl-MA' for PMMA. 
أكثر مواد هذه المجموعة خطورة هى مشتقات سلاسل الدى D-Series فهى الأطول تأثيراً (تستمر لمدة أطول) والأكثر إحداثًا لضيق الشرابين عن باقى المجموعة الفنيثيل أمين(r). وهى تحدث حالات من الهياج، والهلوسة، واضطراب فى ضربات القلب،

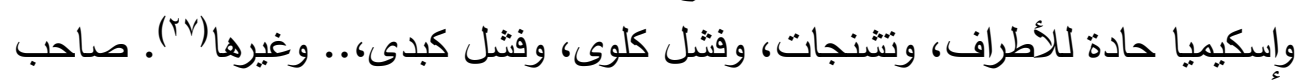

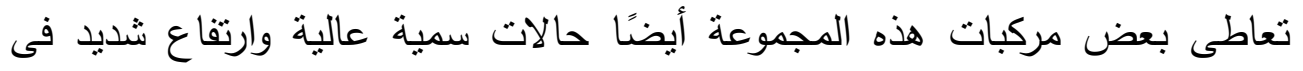

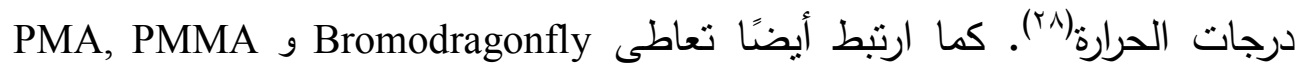
و2C-T-7 من هذه المجموعة بعدد من حالات الوفاة(ra).

رابعًا: همبموعة البيبيبرازين Piperazines سميت هذه المجموعة بالمستحضرات الطبية الفاشلة لأنه كان هناك محاولات كثيرة من شركات الأدوية لتصنيع مستحضرات طبية منها ولكنها كلها باءت بالفشل(·r). فبعض مشتقات هذه المجموعة كانت قد صنعت فى البداية كمضادات للاكتئاب ولكن سرعان ما تم سحبها من الأسواق('آ). عندما وجد أن تأثثرها يماثل تأثثر الأمفيتامينات وأنها تحدث الاعتماد. فى عام ع . . ب تم تصنيع بعض مشتقات من هذه المجموعة

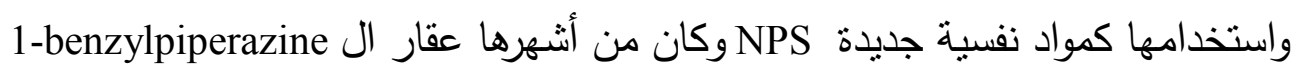
ومشتقات أخرى منل: (BZP) 1-benzylpiperazine (BZP), 1-(3-chlorophenyl) piperazine (mCPP), 1-(3trifluoromethylphenyl) piperazines (TFMPP), 1-benzyl-4-methylpiperazine (MBZP), 1-(4-fluorophenyl) piperazines (pFPP) and 1-cyclohexyl-4-(1,2diphenylethyl) piperazine (MT-45).

(a)<smiles>c1ccc(CN2CCNCC2)cc1</smiles>

(b)<smiles>FC(F)(F)c1cccc(N2CCNCC2)c1</smiles>

(c)

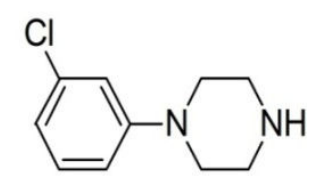

Figure 3: The most common piperazines: (a) 1-benzylpiperazine (BZP),

(b) 1-(3-trifluoromethylphenyl) piperazine (TFMPP) and (c) 1-(3chlorophenyl) piperazine (mCPP) 
وأصبحت نتاع كبديل مشروع (غير خاضع للرقابة) لعقار الاكستاسى

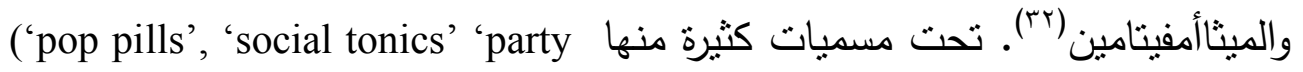
.pills Flying Angel, Legal E or Legal X, and Pep X, Pep L e Jax, A2, Benny ${ }^{(\Gamma)}$ Bear, ove or Nemesis. MCPP is known as 3CPP, 3C1-PP or CPP) ويتوفر عقاقير البيبيرازين فى شكل أقراص غالباً تحمل أثكالاً تشبه الموجودة

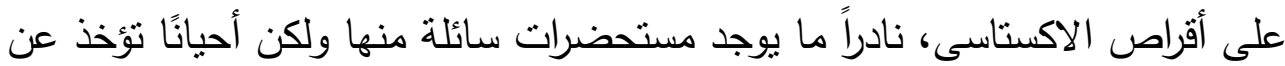
طريق الحقن أو الثم أو التنخين. وعلى الرغم من أن هذه المجموعة من المشتقات غير خاضعة للرقابة الدولية،

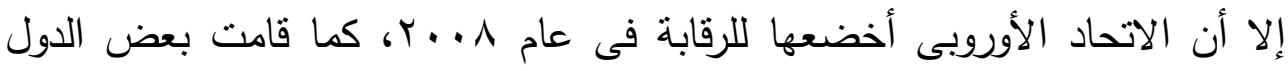

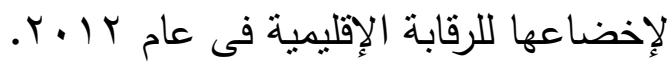

\section{الأضرار الناجمة عن تعاطى هذه المواد}

وجد أن البيبيرازينات تعمل كمنشط للجهاز العصبى من خلال نأثنرها على السيرونونين والدوبامين، والنورادرينالين فى الدخ. وقد تركزت معظم الدراسات على عقار BZP حين ينم تعاطيه منفردا أو مخلوطًا مع TFMPP أو MCPP فوجد أن له وله

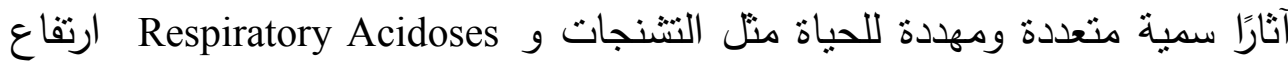

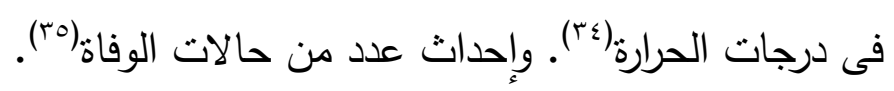

خاهسًا: المواد النفسية البديدة ذات الأصل النباتى Plant-based substances

القات الق Catha Edulis Forsk

شجرة نبات القات شجرة معرة تتمو فى إفريقيا بكينيا والصومال تتبع عائلة كما يزرع على نطاق واسع فى اليمن، ولكنها انتشرت فى الآونة الأخيرة فى البحرين وكندا أو فنلندا وايرلندا والولايات المتحدة وايطاليا ونيوزيلاندا 
والصين حتى أصبحت ثانى عقار بعد ال السليفيا. والمواد الفعالة موجودة فى أوراق

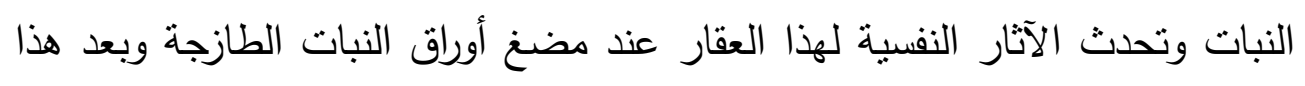

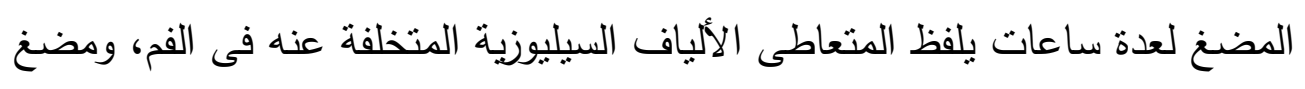

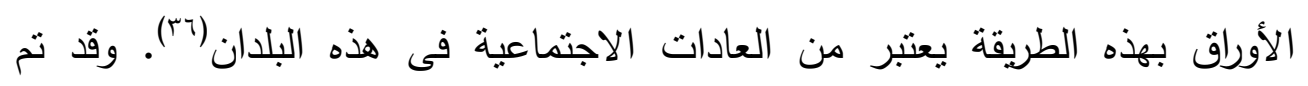

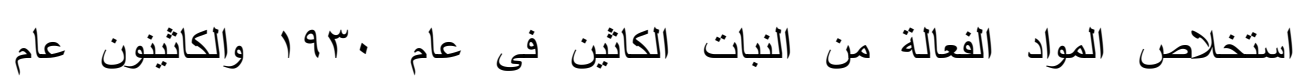
(rv) $)$ 9

qat', 'gat', 'chat', 'miraa', ويحمل القات عدة أسماء شائعة منلك

'murungu' and 'Arabian or Abyssinian tea'

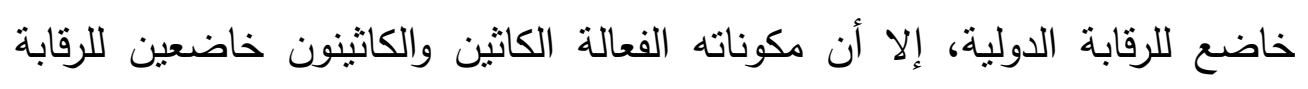
الدولية أما القات فيخضع للرقابة الإقليمية.

\section{آثار تعاطى القات}

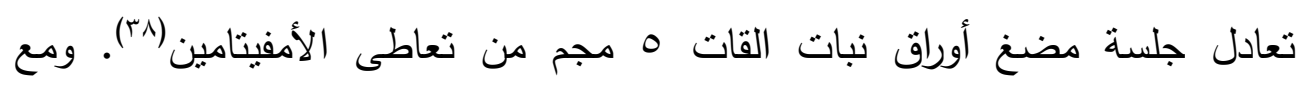

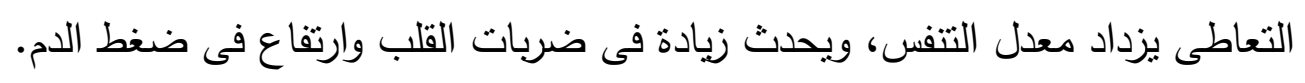

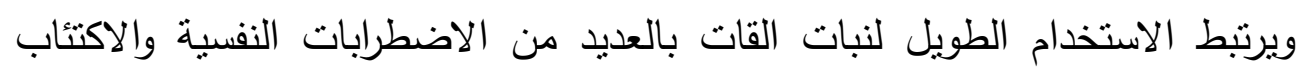

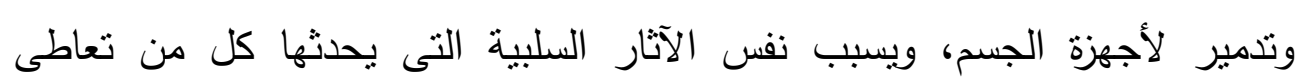

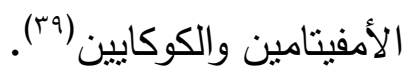

Mitragyna speciosa Korth الكراتوم شجرة الكراتوم شجرة كبيرة تتمو فى المناطق الاستوائية وتحت الاستوائية فى جنوب

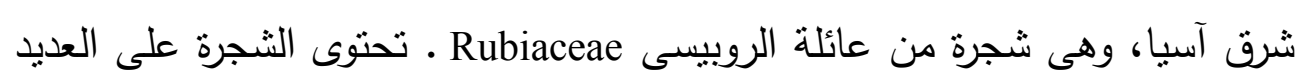

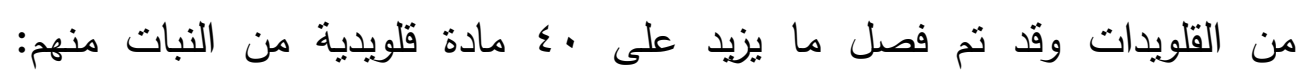

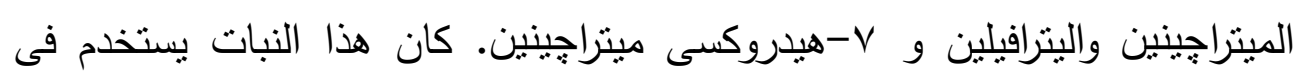




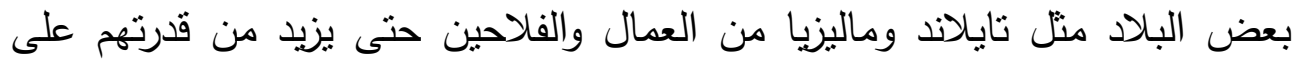

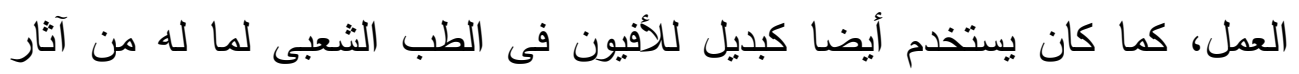

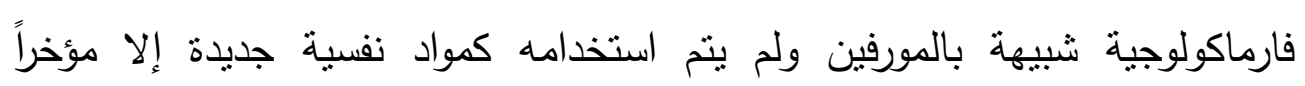

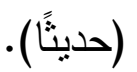

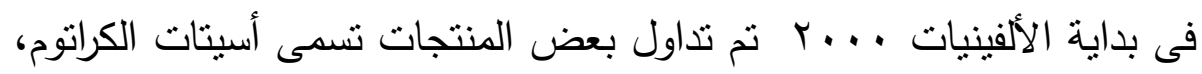

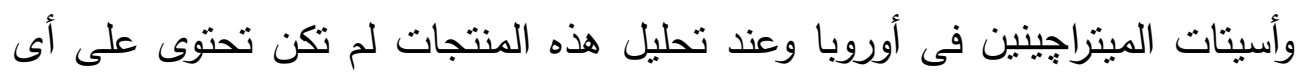

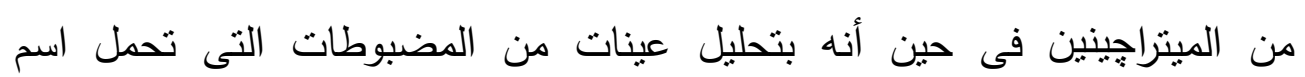
كريبتون (•). وجد أنها نحتوى على الكافيين و O.desmethyl tramadol (أهم نواتج

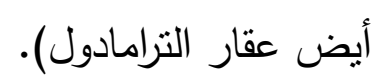
وحديثًا أصبحت تباع بعض المواد النفسية التى تحتوى على الكرانوم تحت اسم

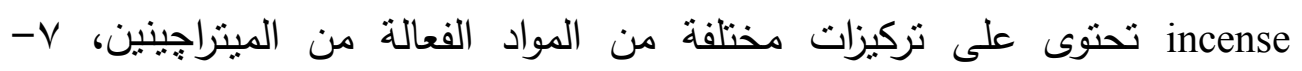

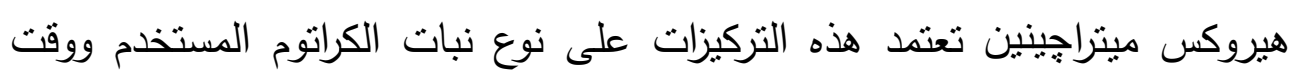
حصناده.

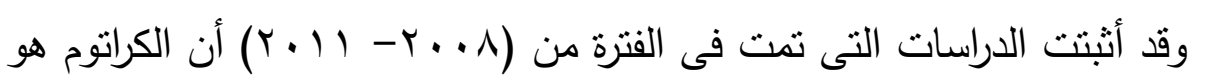

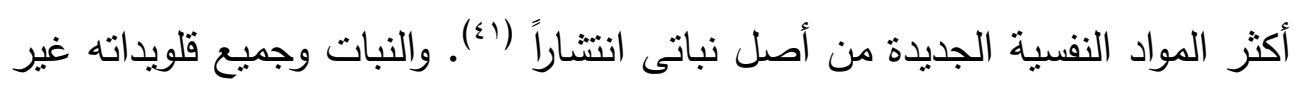

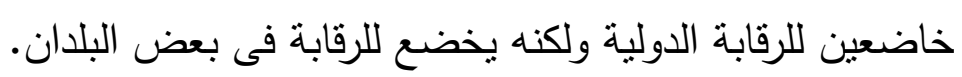

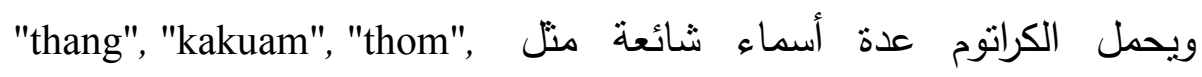
"ketum" and "biak"

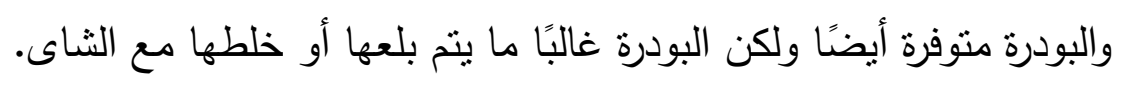

\section{الآثار السلبية لتعاطى الكراتوم}

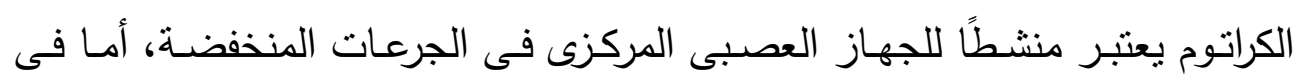

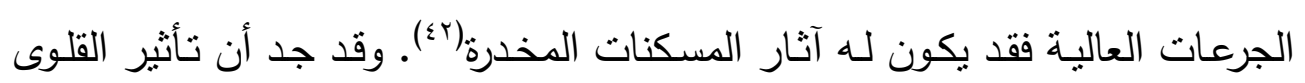


الرئيسى فى النبات وهو الميتراجينين يماثل تأثثير المورفينات، أمـا المشتـق الآخـر

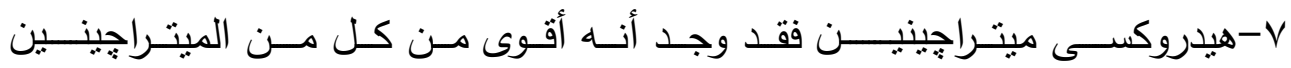

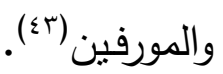

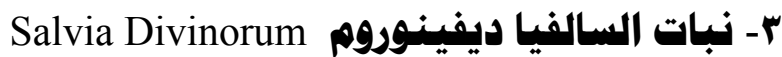

هو نبات من عائلة النعناع لاميسيى (Lamiaceae) وهو من النباتات المؤثرة فى

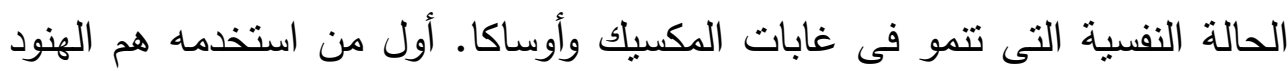

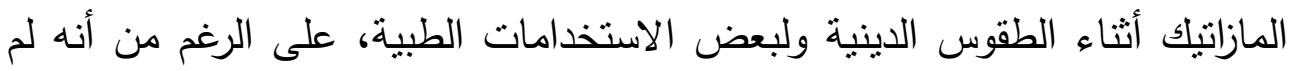

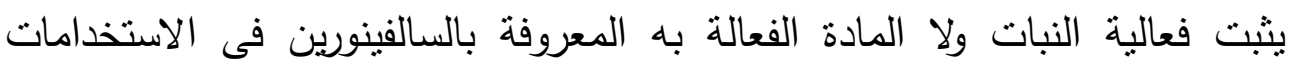

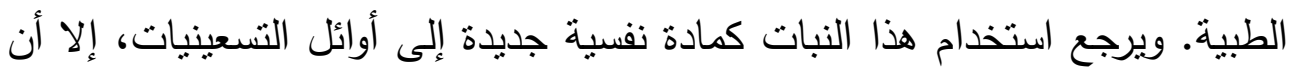

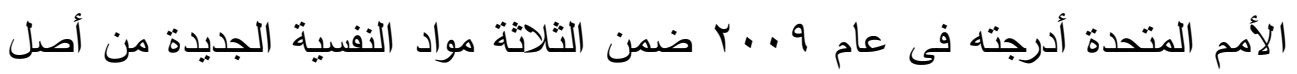
نباتى مع القات والكراتوم. المادة الفعالة فى النبات هى الهى السالفينورين أ (نيوكليرودان

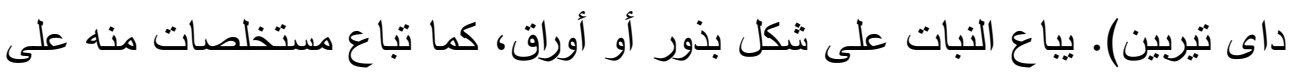

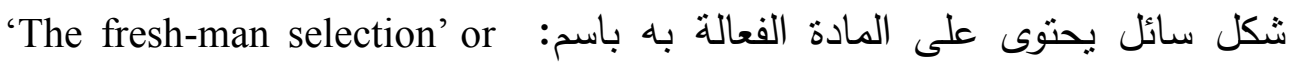
the "starter pack"

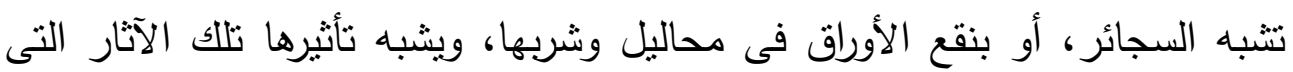

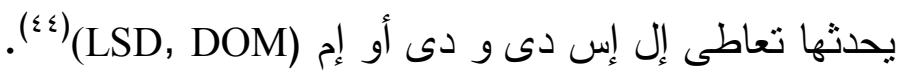

$$
\begin{aligned}
& \text { وللنبات أسماء شائعة كثيرة منها: }
\end{aligned}
$$

"Maria Pastora", "Sage of the Seers", "Diviner’s Sage", "Salvia", "SallyD", "Magic Mint", "Purple Sticky", "Shepherdess's Herb"

نبات السالفيا .(ْ) والمواد الفعالة به السالفينورين غير خاضعين للرقابة الدولية. ونظرًا للانتشار الواسع فى استخدامهم كمواد نفسية جديدة لجأت بعض البلدان لإخضاعهم 


\section{الآثار السلبية لتعاطى السالفيا}

لا توجد دراسات كافية على النبات أو المادة الفعالة به.

\section{سادساً: القنبيات الصنعة Synthetic Cannabinoids}

وهى المواد ذات التركيب المشابه للقنبيات الطبيعية، وهى أكثر المواد النفسية الجديدة المصنعة انتشاراً سواءً فى مصر أو على مستوى العالم ومن أمثلتها: JWH compounds, AB-001, WIN 55,212-2, AM-694, AM-2201, AM-679, RCS-4, CP 47, 497, HU-210, AKB-48 (APINACA), ADB-PINACA, MDMB-CHMICA, TMCP-H, TMCP-018, TMCP-2201, TMCP-200, TMCP-1220， UR-144， 5F-UR-144 (XLR-11)， PB-22， BB-22 (QUCHIC), AB-FUBINACA.

ويؤدى تعاطى هذه المواد إلى كثير من المشاكل الصحية، وعلى الرغم من أن

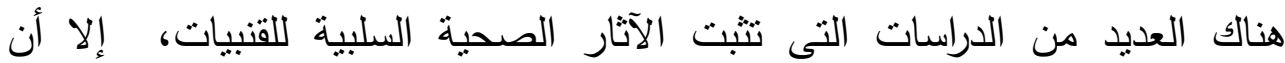

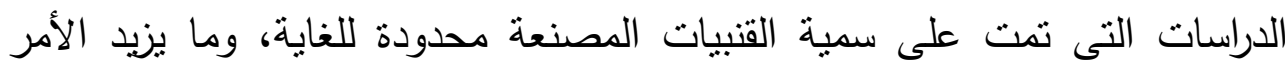

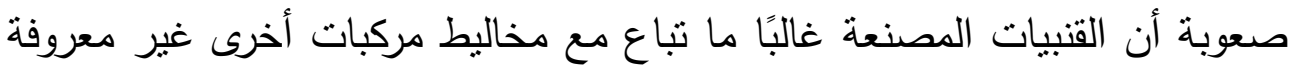
ولكن للأسف آثارها أثند خطورة على صحة المتعاطى من القنبيات الطبيعية. فقد ارتبط تعاطى القنبيات الهصنعة باضطراب فى ضربات القلب، والتشنجات، والاضطرابات النفسية وغيرها، كما ثبت أن بعض نواتج أيض هذه المشتقات له القدرة

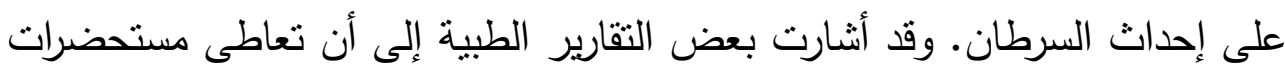

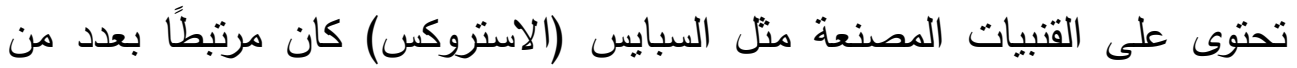

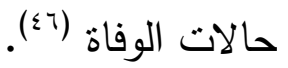


(a)

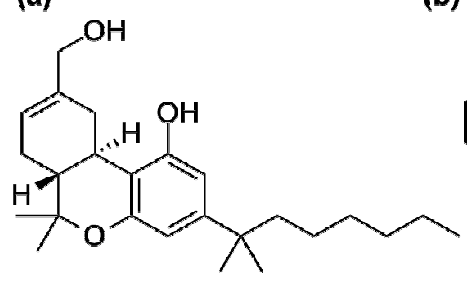

(b)

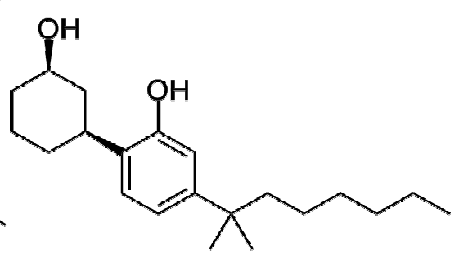

(c)

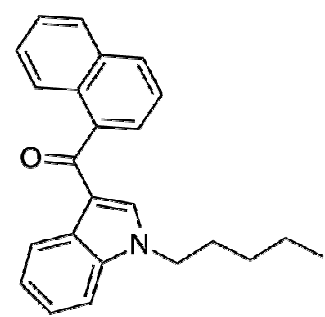

Figure 4: The most common synthetic cannabinoids: (a) HU-210 (classical cannabinoid), (b) CP-47,497 (non-classical cannabinoid), (c) JWH-018 (aminoalkylindoles)

ويؤدى تعاطى القنبيات المصنعة بالإضافة إلى المشاكل الصحية إلى كثير من

المشكلات الاجتماعية والقانونية، لذلك فقد اهتمت بها وزارة الصحة والسكان وأدرجت ست عشرة مادة منها فى جداول المخدرات من خلال ثلاثة قرارات منلاحقة فى عامى

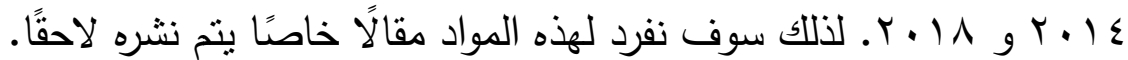

\section{سابها: الكاثينون المسنع Synthetic Cathinones}

الكاثينونات المصنعة هى عبارة بيتا-كيتو فينيثيل أمين وهى تشبه كيميائيًا الأمفيتامين والميثاأمفيتامين. الكاثينون هو المركب الأساسى من المواد الفعالة الموجودة بأوراق نبات القات ويعتبر المركب الرئيسى الذى تصنع منه العديد من الكاثينونات المصنعة. هناك أربع مواد من هذه المجموعة تخضع للرقابة الدولية وهم: الكاثينونوالمينكاتينون - والكانين - والبيروفاليرون.

وقد بدأ ظهور الكاثينون المصنع فى الأسواق فى منتصف الألفينيات، وفى عام 0. . . ظهر الميثايلون وهو شبيه الاكستاسى، تلاه فى عام V . . . ظهور ع - مثيل مينكاثينون (ميفيدرون) فى عدد من الدول(v). حديثًا بدأ فى ظهور مجموعة أخرى من الكاثينون المصنع فى الأسواق شبيهة بالبيروفاليرون (r-ع ميثنلين داى أوكسى بيروفاليرون (MDPV)، والنافيرون الذى أدرج ضمن المواد النفسية الجديدة فى عام 


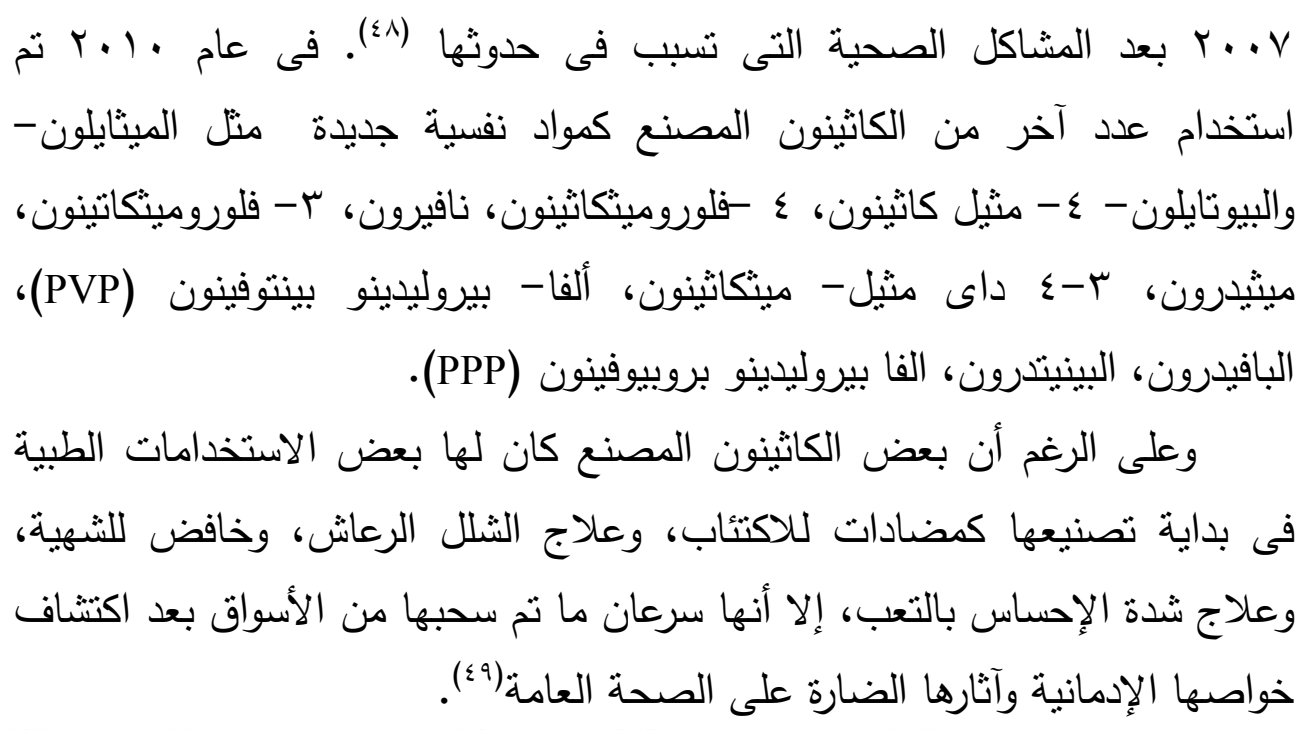

(a)<smiles>CC(N)C(=O)c1ccccc1</smiles>

(d)<smiles>CCCC(C(=O)c1ccc(C)cc1)N1CCCC1</smiles>

(b)<smiles>CNC(C)C(=O)c1ccc(C)cc1</smiles><smiles>CCCC(C(=O)c1ccc2c(c1)OCO2)N1CCCC1</smiles>

(c)<smiles>CNC(C)C(=O)c1ccc2c(c1)OCO2</smiles><smiles>CCCC(C(=O)c1ccc2ccccc2c1)N1CCCC1</smiles>

Figure 5: Synthetic cathinones: (a) cathinone, (b) 4-methylmethcathinone (mephedrone), (c) methylone,(d) pyrovalerone,

(e) 3,4methylenedioxypyrovalerone, (f) naphyrone

$$
\text { وبياع الكاثينون المصنع بأسماء: }
$$

'research chemicals', 'plant food', 'bath salts' or 'glass cleaner'. Mephedrone ('m-cat', 'meph', 'drone' or 'miaow') and methylone ('explosion' or 'top cat') are usually available as white or brown powders or in the form of pills that are often sold as 'ecstasy'. Mephedrone is commonly nasally insufflated, injected, ingested by swallowing a powder wrapped in paper (bombing), or mixed in a drink. 
وغالبًا ما يباع الكاثينون المصنع فى شكل أقراص أو كبسولات أو على شكل

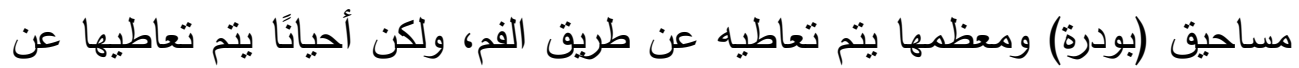

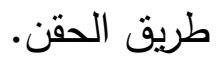

ويعمل الكاثينون الدصنع على تتشيط الجهاز العصبى المركزى من خلا التأثنر على كل من الموصلات العصبية الدوبامين والنورابينيفرين والسيروتونيتين، يماتل فى ذلك المخدرات التقليدية الكوكايين والاكستاسى والميثامفيتامين.

\section{الآثار الصحية لتعاطى الكاثينون المنع}

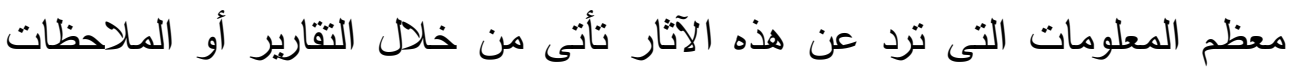

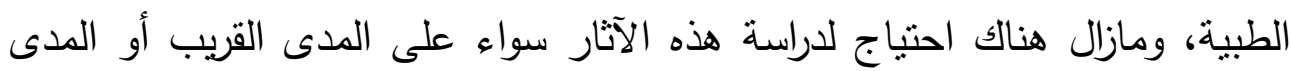

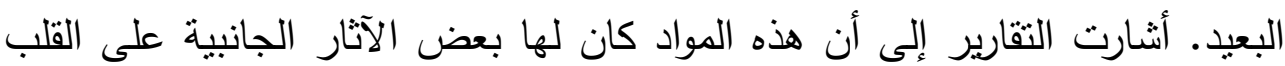

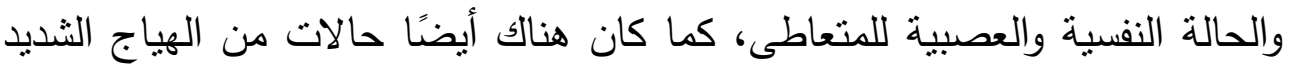

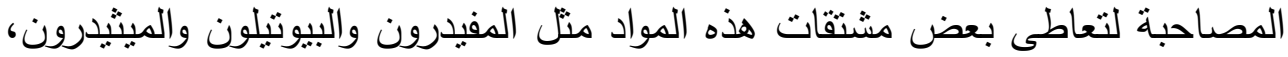

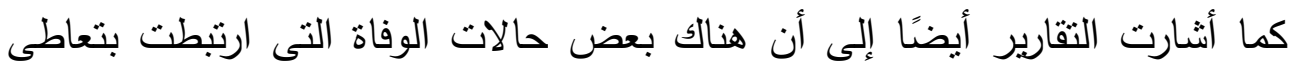

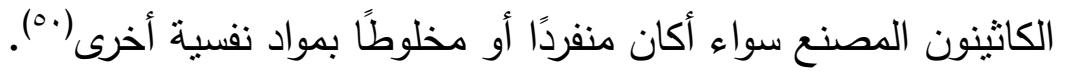

\section{Tryptamine ثاهنًا: هشتقات التزيبتاهين}

مشتقات التزيبتامين التى تتدرج تحت المواد النفسية الجديدة هى مركبات مشتقة من

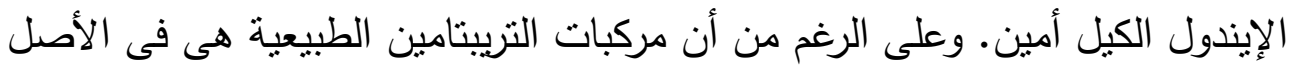

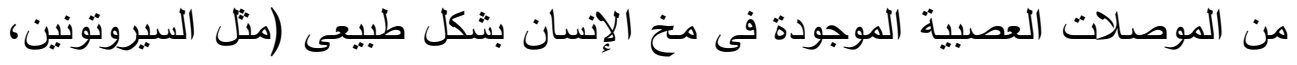

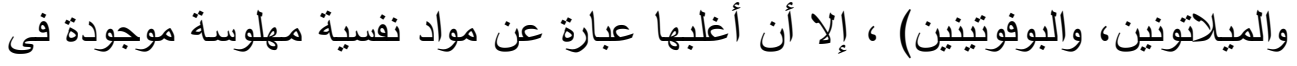

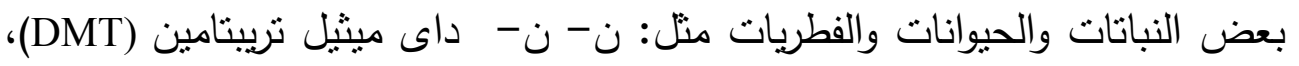

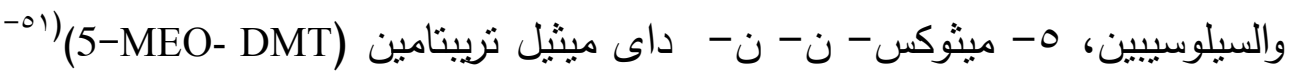


وقد ظهر حديثًا فى الأسواق مجموعة من التريبتامين المصنع من DMT، ومن

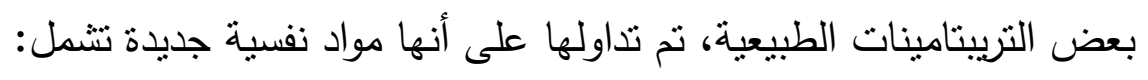
. 5-MEO-DMT, 5- MEO DPT, AMT, 4-AcO- DMT and, 4-AcO- DiPT

(a)

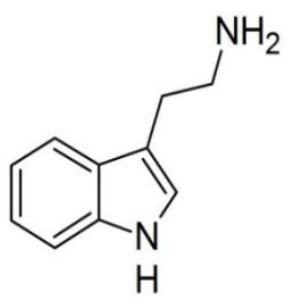

(b)

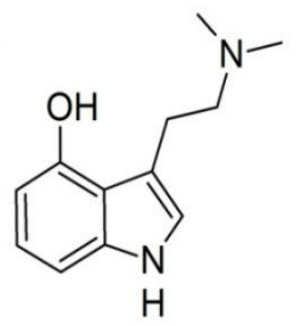

(c)

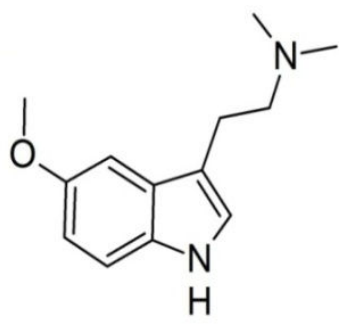

Figure 6: Tryptamine and related compounds: (a) tryptamine, (b) psilocin and (c) 5'-methoxy-N,N-dimethyltryptamine (5-MeO-DMT) والجدير بالذكر أن السيلوسين والسيلوسيبين فقط يخضعان للرقابة الدولية أما باقى المشتقات فهى تخضع فقط للرقابة الإقليمية.

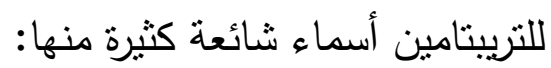

Foxy-Methoxy' (5-MeO-DIPT); 'alpha-O', 'alpha' and 'O-DMS' (5-MeOAMT); '5-MEO' (5-MeO-DMT).

تباع مشتقات التريبتامين المصنع الجديدة كأقراص أو كبسولات، أو على شكل

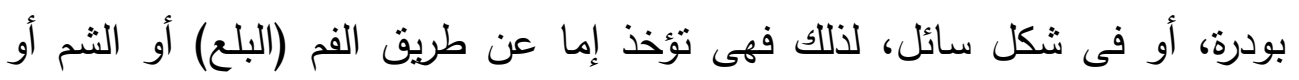
الحقن.

تعمل مشتقات التريبتامين كمواد مسببة للهلوسة من خلال تأثيرها على بعض مستقبلات السيروتونين وتحدث الهلوسة. مركبات هذه المجموعة تنبه المخدرات التقليدية المسببة للهلوسة المعروفة منل DMT وعقار الهلوسة LSD، و و 2C-B. ولكنها أحيانا يكون لها تأثثر منشط. 
أثتتت الدراسات أن تعاطى مركبات هذه المجموعة له آنار جانبية متعددة منها: الشعور بعدم الارنياح، حالة من الهياج، اضطرابات فى الجهاز الهضمى، وتوتز

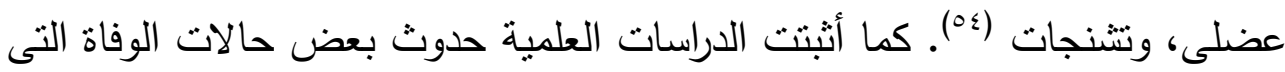

صاحبت تعاطى بعض مشتقات التربيتامين منل الFOXY وغيرها (00).

(a)

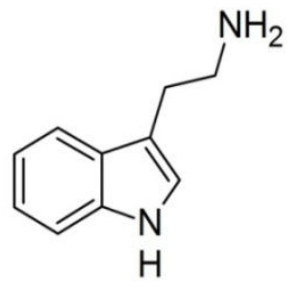

(b)

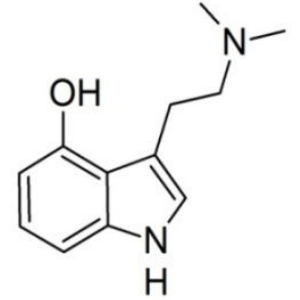

(c)<smiles>COc1ccc2[nH]cc(CCN(C)C)c2c1</smiles>

Figure 7: Tryptamine and related compounds: (a) tryptamine, (b) psilocin and (c) 5'-methoxy-N,N-dimethyltryptamine (5-MeO-DMT).

\section{تاسعاً: هواد نفسية جديدة هتنوعة أخرى}

هى مجموعة من المواد النفسية الجديدة NPS التى يساء استخدامها وهى متتوعة من ناحية التركيب الكيميائى لكنها لا تتبع أى من المجموعات السابقة ، ولكن آثارها الفارماكولوجية تشبه تللك الآتار التى تحدثها عقاقير الهلوسة والأفيونيات المصنعة (الفينتانيل)، والمسكنات المنومة، والمنشطات وقد يدخل تحت هذه المجموعة مجموعة البنزوديازبيينات المصنعة الجديدة. على الرغم من أن مجموعة عقاقير البنزوديازيبينات لها أهمية كبيرة فى المجال الطبى وتستخدم على نطاق واسع فى العلاج، إلا أن آثارها الإدمانية والاعتماد عليها أثنار الاهتمام على المستوى العالمى خاصة مع الإفراط الثديد فى استخدامها فى الوصفات الطبية ولسهولة الحصول عليها. وقد تم تصنيع مجموعة 
من مشتقات البنزوديازيينات كمواد نفسية جديدة وأصبحت تباع على الإنترنت

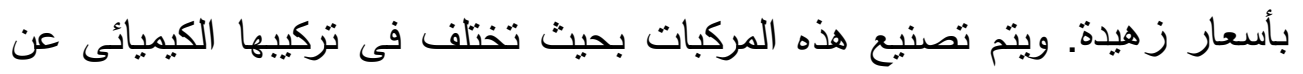

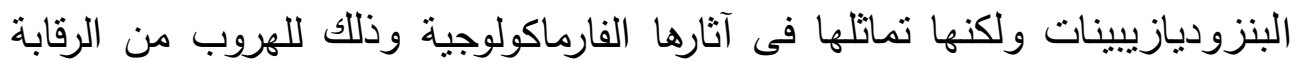
الإقليمية والدولية عليها، لذلك فهى تسمى أحيانًا ب. "Designer Benzodiazepines"

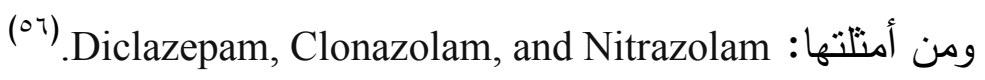
ويتم تصنيعها بطريقتين إما بعمل تعديل بسيط فى تركيب بعض العقاقير

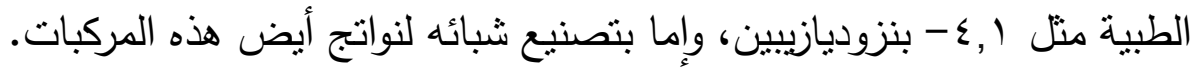

\section{الآثار الصحية الناجمة عن تعاطى المواد النفسية الجديدة (NPS)}

هذا بالإضافة إلى أن هناك اختلافات فردية فى الاستجابة لتأثثر هذه المواد، كما أن

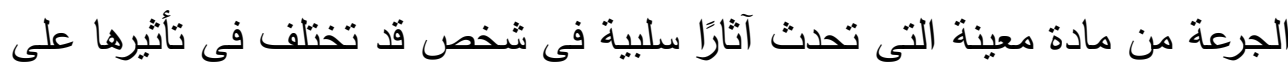
شخص آخر. على الرغم من أن بعض الآثار نرتبط بالمجموعة التى ينتمى إليها

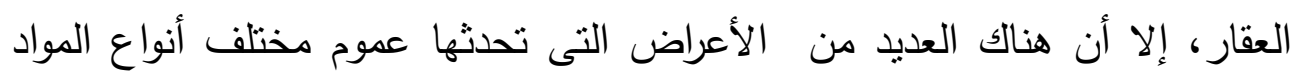
النفسية الجديدة بشكل عام يمكن تلخيصها فى الآتى (ov):

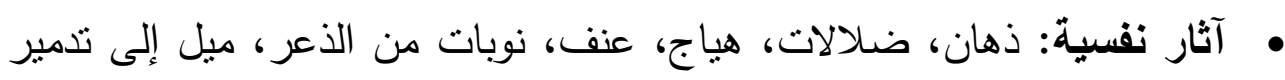

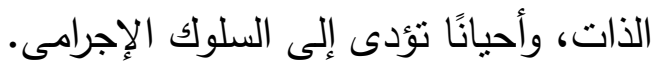

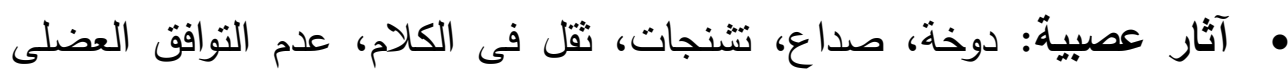
العصبى، اضطراب النوم أرق وكوابيس، عدم وضوح الروئية.

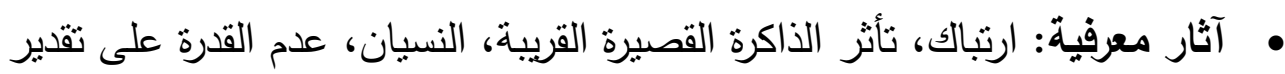
الزمن والمسافات، اضطراب ذهنى.

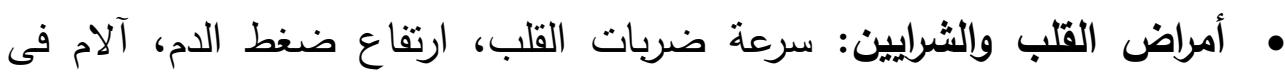

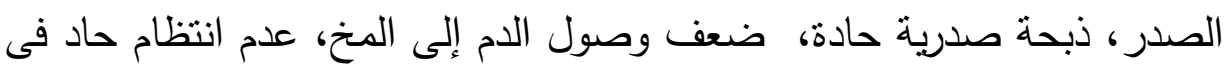

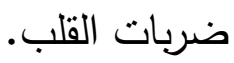


• أعراض متنوعة: دوخة، غثيان، قىء، آلام فى العضلات، اضطراب حاد فى إلى

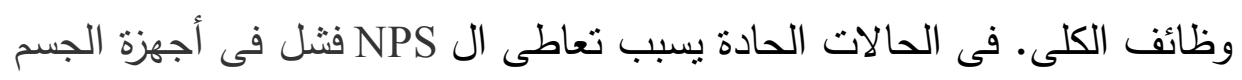
اليختلفة، أزمة قلبية، هبوط حاد فى التنفس والوفاة.

\section{الوضع القانونى للمواد النفسية الجديدة}

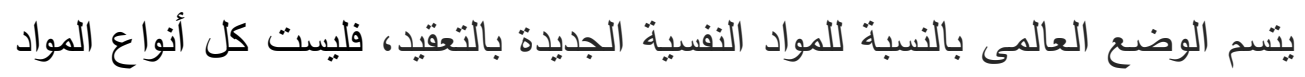

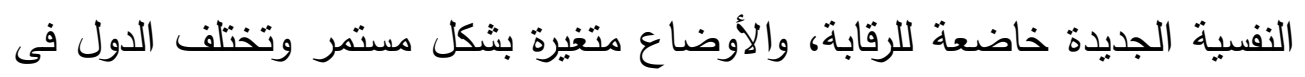

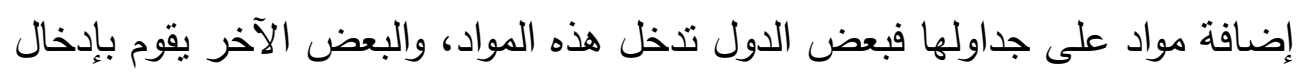

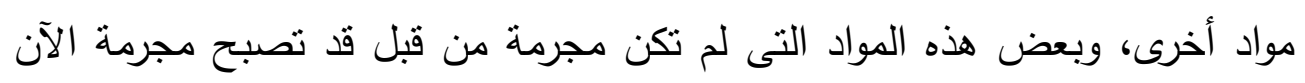
فالوضع متغير بشدة في الآونة الأخيرة.

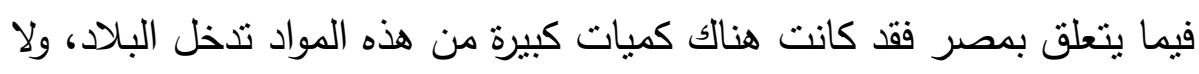

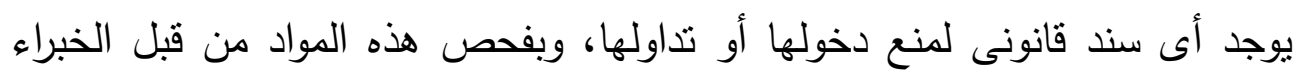

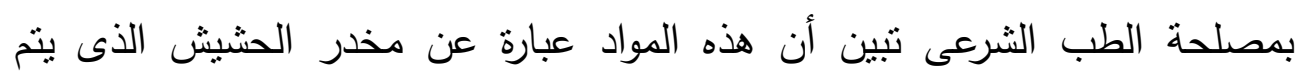

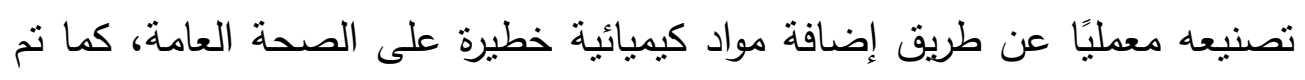
تعيين الثوابت القياسية لها. وأضافت أن المواد المخدرة التى تم إدراجها تحمل أسماء

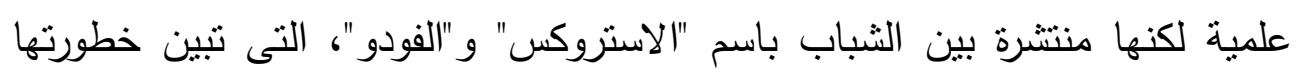

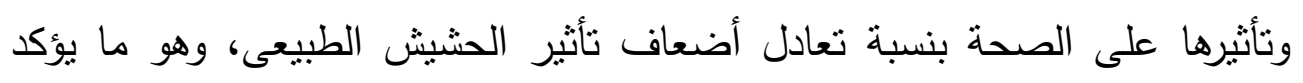

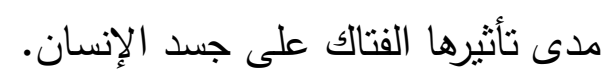

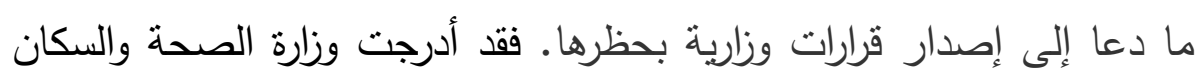
مخدرات "الإستروكس" و "الفودو" والتنى أصبحت من أكثر المواد المسيبة للإدمان

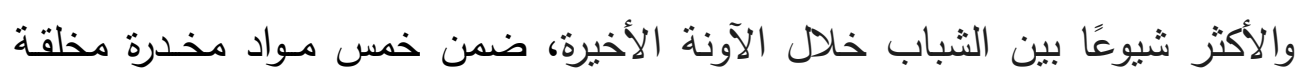

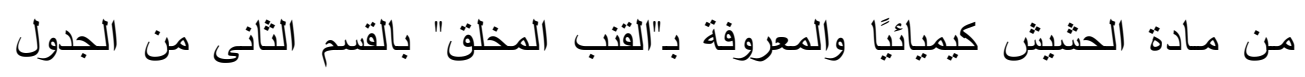




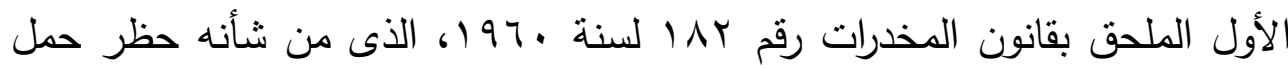

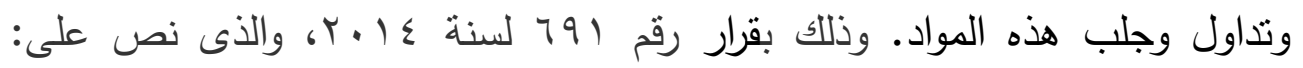
إدراج خمس مواد مخدرة من أنواع الحشيش الصناعى وأملاحها ونظائرها وإستراتها

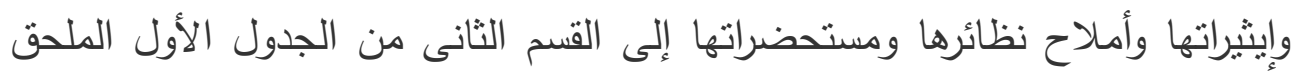

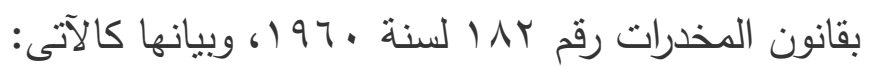

1-Penty1-3-(1-naphthoy1) indole (JWH-018).

1-buty1-3-(1-naphthoy1) indole (JWH-073).

1-\{2-(4-morpholiny1) ethy1\} -3-(1-naphthoyl) indole (JWH-200).

5-\{1,1-(dimethylhepty1)-2-(3-hydroxycyclohexyl)-phenol (CP-47,497).

5-(1,1-dimethyloctyl)-2-(3-hydroxycyclohexyl)-phenol (cannabicyclohexanol, CP-47, 497 C8 homologue)

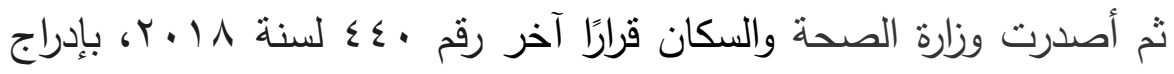

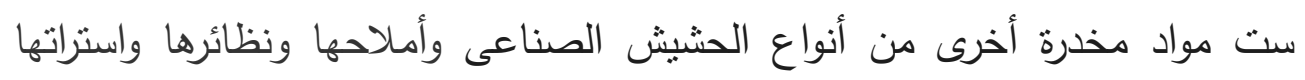

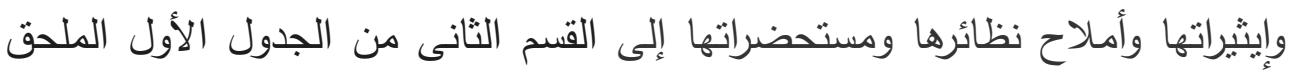

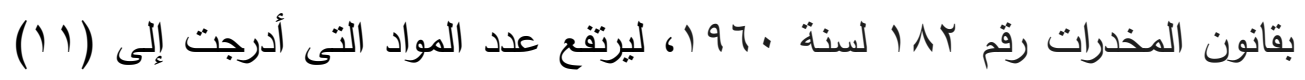
مادة. هذه المواد هى:

1- AB-Fubinaca:.n-[(2s)-1-amino-3-methyl-1-oxobutan-2-yl]-1-[(4-fluorophenyl)methyl]indazole-3-carboxamide.

2- AB-Chminaca: n- [(2s)-1-amino-3-methyl-1-oxobutan-2-yl]-1-(cyclohexylmethyl)indazole-3-carboxamide.

3- XLR-11: (1-(5-fluoropentyl)-1H-indol-3-yl)(2,2,3,3-tetramethylcyclopropyl)methanone.

4- XLR-11N-(4-fluoropentyl) isomer: (1-(4-fluoropentyl)-1H-indol-3-yl) (2,2,3,3-tetramethylcyclopropyl)methanone.

5- FUB-AMB: methyl (s)-2-[1-(4-fluorophenyl)methyl]Indazole-3-methylbutanoate.

6- 5-fluoro ADB: methyl (s)-2-[1-(5-fluoropentyl)-1h-indazole-3-carboxamido]-3,3-dimethylbutanoate. 


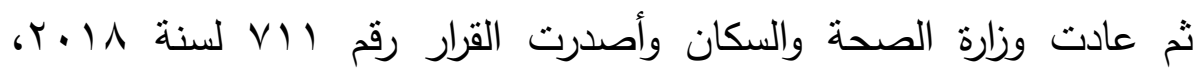
والخاص بإضافة خمس مواد أخرى ومستحضراتها أينما وجدت إلى القسم الثانى من ولن

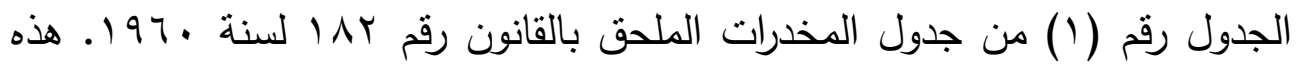

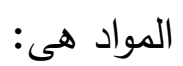

1- SDB-005: naphthalen-1-yl 1-pentyl-1H-indazole-3-carboxylate.

2- EMB -Fubinaca:ETHYL \{1-(4-Fluorobenzyl)-1H-indazole-3-Carbonyl\} -L-Valinate.

3- 5-FLUORO PY - PICA: $\{1$ - (5-fluoropentyl) - 1H -Indol -3-YL $\}$ \{Pyrrolidin - 1 - YL\} Methanone.

4- ETHCATHINONE: 2 - Ethylamino - 1 - Phenyl - Propan - 1-One.

5-4- CHLOROETHCATHINON: 1- (4-CHLOROPHENYL)-2-(Ethylamino) Propan -1-One.

مع تعديل المركب الخـامس المذكور بالهـادة (1) من القرار الوزارى رقم •؟؟

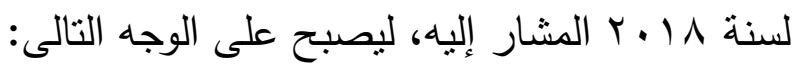
5-FYB - AMB (AMB - Fubinaca) (MMB - FUBINACA) methyl (2S) -2$\{1-(\{4-$ fluorophenyl) methyl $\}$ indazole $-3-$ carbonyl) amino $\}$-3methylbutanoate.

نظرًا لما شهدته السنوات الأخيرة من نوليف الأعداد الهائلة من المواد النفسية الجديدة (NPS)، التى تصدرت سوق المخدِّات الاصطناعية والجديد منها الذى يدخل تباعًا؛ وعلى الرغم من اختلاف أنماط التعاطى بين مختلف الدول، إلا أن المشترك بينها أن الن الناعل القنبيات المصنعة والكاثينونات المصنعة هم الأكثر انتشارًا على المستوى العالمى.

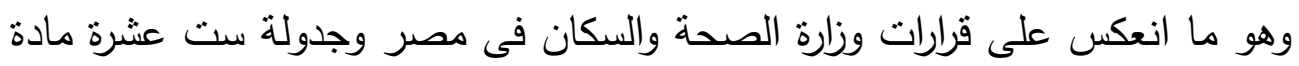

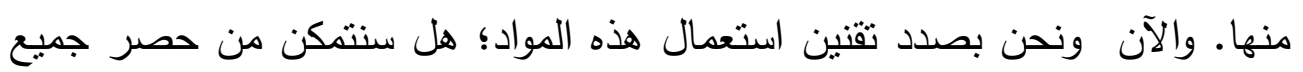
هذه الأعداد التى دخلت والتى سوف تدخل تباعًا إلى الأسواق الدصرية ونستصدر بها

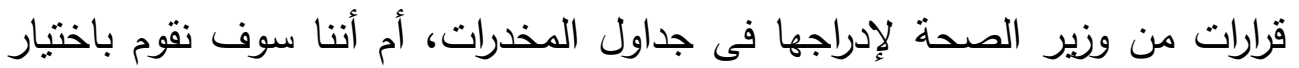


الأكثر انتثارًا منها فى مصر أو ريما الأكثر خطورة، أم أننا سوف نكتفى بما تم إلحاقه بجداول المخدرات العالمية.

فى تصورى أننا نحتاج إلى رصد للمواد النفسية الجديدة بكافة أنواعها، والتعرف

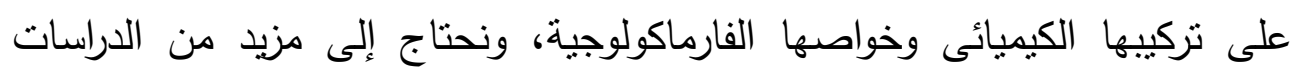

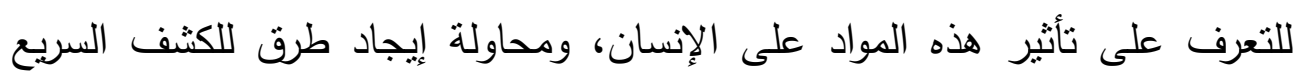

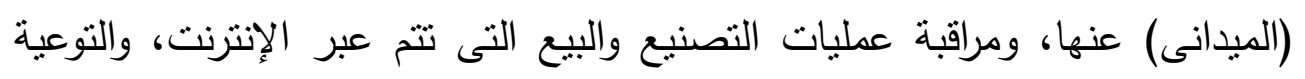

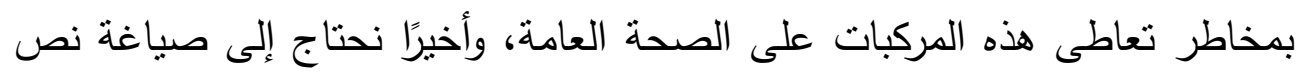

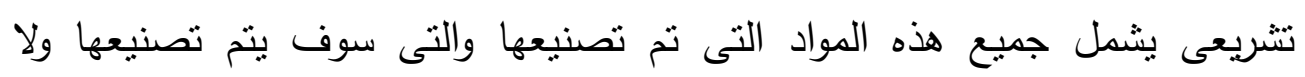

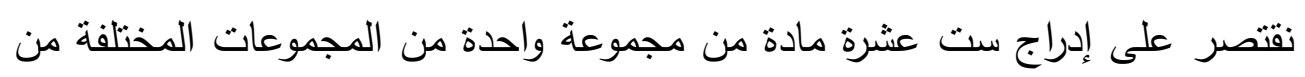

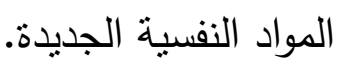




\section{المراجع}

1- 1-UNODC. United Nations Office on Drugs and Crime, World Drug Report 2018.

2- Corazza O, Demetrovics Z, van den Brink W, Schifano F. "Legal highs" an inappropriate term for "Novel Psychoactive Drugs" in drug prevention and scientific debate, Int J Drug Policy. 2013; 24(1):82-83.

3- http://english.ahram.org.eg/NewsContent/1/64/311917/Egypt/Politics-/Voodoo-Egyptsbattle-against-Satans-drug.aspx.

4- Schifano F, Orsolini L, Duccio Papanti G, Corkery JM. Novel psychoactive substances of interest for psychiatry. World Psychiatry. 2015;14(1):15-26. doi: 10.1002/wps. 20174.

5- UNODC. United Nations Office on Drugs and Crime, World Drug Report 2013, Vienna, [cited 2016 May 5], from: https:/www.unodc.org/unodc/secured/wdr/wdr2013/ World_Drug_Report_2013.

6- Clinical Commission 2011; Farré 2011; Hill 2011; EMCDDA 2012; EMCDDA 2013; UNODC 2013; Prosser 2011.

7- Julia González Alonso and Noelia Llorens Aleixandre, NEW PSYCHOACTIVE SUBSTANCES. A CHALLENGE TO PUBLIC HEALTH, The Spanish Early Warning System, SERVICIOS ADI EDITORIALES, http://www.pnsd.mscbs.gob.es/profesiona, Legal deposit: M-21193-2014, ISBN: 978-84-940849-5-9.

8- Abdulrahim D, Bowden-Jones O, on behalf of the NEPTUNE Expert Group, 2015, Guidance on the management of acute and chronic harms of club drugs and novel psychoactive substances. http://neptune-clinicalguidance. March-2015. pdf. Accessed 24 Apr 2017.

9- https://www.unodc.org/LSS/SubstanceGroup/GroupsDashboard?testType=NPS

10-E. Solomons and J. Sam, "2-aminoindans of pharmacological interest", Journal of Medicinal Chemistry 16, 1973: 1330-33.

11-A.P. Monte, D. Marona-Lewicka, N.V. Cozzi and D.E. Nichols, "Synthesis and pharmacological examination of benzofuran, indan, and tetralin analogs of 3,4(methylenedioxy)amphetamine", Journal of Medicinal Chemistry 36, 1993: 3700-6.

12-P.D. Sainsbury, A.T. Kicman, R.P. Archer, L.A. King and R.A. Braithwaite "Aminoindanes - the next wave of 'legal highs'?", Drug Testing and Analysis 3, 2011: 479-482.

13-P.V. Kavanagh, J. Sharma, S. McNamara, D. Angelov, S. McDermott, D. Mullan and S. Ryder, Head shop 'legal highs' active constituents identification chart (May 2010, pre-ban). 
14-H.M. Connolly, J.L. Crary, M.D. McGoon, D.D. Hensrud, B.S. Edwards, W.D. Edwards and H.V. Schaff, "Valvular heart disease associated with fenfluraminephentermine", New England Journal of Medicine 337 (1997): 581-8.

15-Baldridge, E.B., Bessen, H.A., 'Phencyclidine', Emergency Medicine Clinics of North America, 1990, 8 (3), 541-50; Balster, R.L., 'The behavioral pharmacology of phencyclidine', in H.Y. Meltzer (Eds.), Psychopharmacology: The third generation of progress, New York, 1987, 1573-9; The structure-activity relationships among arylcycloalkylamines can be further consulted in Manallack, D.T., Davies, J.W., Beart, P.M., Saunders, M.R. and Livingstone, D.J., 'Analysis of the biological and molecular properties of phencyclidine-like compounds by chemometrics', Arzneimittelforschung, 1993, 43 (10), 1029-32

16-Pearlson, G.D., 'Psychiatric and medical syndromes associated with phencyclidine (PCP) abuse', Johns Hopkins medical journal, 1981, 148, 25-33; Smith, J.B., 'Situational specificity of tolerance to effects of phencyclidine on responding of rats under fixed-ratio and spacedresponding schedules', Psychopharmacology, 1991, 103, $121-8$.

17-World Health Organization, Model List of Essential Medicines: 19th List (April 2015) amended November.

18-United Nations Office on Drugs and Crime, 'UNODC questionnaire on new psychoactive substances', submitted by Member States and a network of drug analysis laboratories in 2012.

19-World Health Organization, Ketamine (INN) Update Review Report, Agenda Item 6.1. Expert Committee on Drug Dependence. Thirty-seventh Meeting. Geneva, 16 to 20 November 2015.

20-Gorelick, D.A. and Balster, R.L., 'Phencyclidine (PCP)', in F.E. Bloom \& R.L. Kupfer (Eds.), Psychopharmacology: The fourth generation of progress, New York, 1995, 1767-76; Brecher, M., Wang B.W., Wong, H. and Morgan, J.P., 'Phencyclidine and violence: clinical and legal issues', Journal of Clinical Psychopharmacology, 1988, 8 (6), 397-401; Daghestani, A.N. and Schnoll, S.H., 'Phencyclidine abuse and dependence', Treatments of Psychiatric Disorders: A task force report of the American Psychiatric Association, American Psychiatric Association, Washington D.C., 1989, 1209-18.

21-Hill, SL., Thomas, S.H., 'Clinical toxicology of newer recreationaldrugs', Journal: Clinical Toxicology, 2011, 49(8), 705-19.

22-European Monitoring Centre for Drugs and Drug Addiction, 'Report on the risk assessment of PMMA in the framework of the joint action on new synthetic drugs', 2003, 56 .

23-Huang, H.H. and Bai, Y.M. 'Persistent psychosis after ingestion of a single tablet of '2C-B', Journal: Progress in Neuro-Psychopharmacology \& Biological Psychiatryis, 2010, 35 (1), 293-4. 
24-Monte, A.P., Waldman, S.R., Marona-Lewicka, D., Wainscott, D.B., Nelson, D.L., Sanders-Bush, E., Nichols, D.E., 'Dihydrobenzofuran analogues of hallucinogens. 4. Mescaline derivatives', Journal of Medicinal Chemistry, 1997, 40 (19), 2997-3008.

25-Collins, M., 'Some new psychoactive substances: precursor chemicals and synthesisdriven end-products', Drug Testing and Analysis, 2011, 3 (7-8), 404-16.

26-Hill, S. and Thomas S. H., 'Clinical toxicology of newer recreational drugs', Clinical Toxicology, 2011, 49, 705-19.

27-King's College London, Institute of psychiatry, Psychonaut Web Mapping Research Group, 'Bromo-Dragonfly report', London UK, 2009, (http://194.83.136.209/ documents/reports/Bromodragonfly. pdf; accessed in: September 2012.

28-Ling, L.H., Marchant, C., Buckley, N. A., Prior, M., Irvine, R.J., 'Poisoning with the recreational drug paramethoxyamphetamine (death), Medical Journal of Australia, 2001, 174, 453-55; Elliot, S.P., 'Fatal poisoning with a new phenethylamine: 4methylthioamphetamine (4-MTA)', Journal of Analytical Toxicology, 2000, 24, 85-9; Lamberth, P.G., Ding, G.K., Nurmi, L.A., 'Fatal paramethoxy-amphetamine (PMA) poisoning in the Australian Capital Territory', Medical Journal of Australia, 2008, 188, 426.

29-Andreasen, M.F., Telving, R., Birkler, R., Schumacher, B. and Johannsen, M., 'A fatal poisoning involving Bromo-Dragonfly', Annales de Toxicologie Analitique, 20 (1), 155; Personne, M., Hulten, P., 'Bromo-Dragonfly, a life threatening designer drug', Journal: Clinical Toxicology, 2008, 46, 379-80

30-King, L.A and A.T. Kicman, "A brief history of "new psychoactive substances", Drug Testing Analysis 3, 2011: 401-403.

31-European Monitoring Centre for Drugs and Drug Addiction (EMCDDA), "EMCDDA Risk Assessments: Report on the risk assessment of BZP in the framework of the Council decision on new psychoactive substances", Germany, 2009.

32-Approximately 1.5 to 2 million tablets had been manufactured by Vitafit Nutrition Ltd. for Stargate International (one of the major distributors in New Zealand) since 2001" in "The Expert Advisory Committee on Drugs (EACD) advice to the Minister on: Benzylpiperazine (BZP)", New Zealand, April 2004; Industry figures point out that 26 million doses were sold over an 8-year period; Stargate International, "Party pills: successful safety record", Press Release, 13 March 2008.

33-Drug Enforcement Administration (DEA), Office of Diversion Control, "NBenzylpiperazine. (Street Names: BZP, A2, Legal E or Legal X)", March 2014; European Monitoring Centre for Drugs and Drug Addiction (EMCDDA), "EMCDDA Risk Assessments: Report on the risk assessment of BZP in the framework of the Council decision on new psychoactive substances", Germany, 2009; World Health Organization (WHO), "N-benzylpiperazine (BZP): Pre-Review Report", Expert Committee on Drug Dependence, Thirty-fifth Meeting", Hammamet, Tunisia, 4-8 June 2012. 
34-Gee, P., Jerram, T. and Bowie, D., "Multiorgan failure from 1-benzylpiperazine ingestion-legal high or lethal high?", Clinical Toxicology 48, 2010: 230-3.

35-Elliott, S., "Current awareness of piperazines: pharmacology and toxicology", Drug Testing and Analysis 3, 2011: 430-8; for more information on fatalities related to BZP see World Health Organization (WHO), "N-benzylpiperazine (BZP): Pre-Review Report", Expert Committee on Drug Dependence, Thirty-fifth Meeting”, Hammamet, Tunisia, 4-8 June 2012.

36-Sawair, F.A., Al-Mutwakel, A., Al-Eryani, K., Al-Surhy, A., Maruyama, S., Cheng, J., Al-Sharabi, A. and Saku, T., 'High relative frequency of oral squamous cell carcinoma in Yemen: qat and tobacco chewing asits aetiological background', International Journal of Environmental Health Research, 2007, 17, 185-95.

37-European Monitoring Centre for Drugs and Drug Addiction, 'khat', Drug Profiles (www.emcdda.europa.eu).

38-Dhaifalah I. and Santavy J., 'Khat habit and its health effect. A natural amphetamine', Biomedical Papers, 2004, 148, 11-5.

39-Hoffman, R. and Al'absi, M., 'Khat use and neurobehavioural functions: suggestions for future studies', Journal of Ethnopharmacology, 2010, 132, 554; Morrish, P.K., Nicolaou, N., Brakkenberg, P. and Smith, P.E., 'Leukoencephalopathy associated with khat misuse', Journal of Neurology, Neurosurgery, and Psychiatry, 1999, 67, 556; Odenwald, M., 'Chronic khat use and psychotic disorders: a review of the literature and future prospects', Sucht, 2007, 53, 9-22.

40-European Monitoring Centre for Drugs and Drug Addiction, 'kratom', Drug Profiles (www.emcdda.europa.eu).

41-op. cit.

42-op. cit.

43-Kikura-Hanajiri, R., Kawamura, M., Maruyama, T., Kitajima, M., Takayama, H. and Goda, Y., Simultaneous analysis of mitragynine, 7 hydroxymitragynine, and other alkaloids in the psychotropic plant "kratom" (Mitragynaspeciosa) by LC-ESI-MS', Forensic Toxicology, 2009, 27 (2), 67-74.

44-European Monitoring Centre for Drugs and Drug Addiction, 'Salvia divinorum', Drug Profiles (www.emcdda.europa.eu)

45-United States, Drug Enforcement Administration, 'Salvia divinorum and salvinorin A', 2012. (http://www.deadiversion.usdoj.gov/drugs_concern/salvia_d.pdf); European Monitoring Centre for Drugs and Drug Addiction, 'Salvia divinorum', Drug Profiles (www.emcdda.europa.eu).

46-Ludger, E., et.al., "Synthetic cannabinoids in 'spice-like' herbal blends: first appearance of JWH-307 and recurrence of JWH-018 on the German market", Forensic Science International 222.1 , 2012: 216-222. 
47-Kelly, J.P. "Cathinone derivatives: A review of their chemistry, pharmacology and toxicology", Drug Testing and Analysis 3, 2011: 439-453.

48-European Monitoring Centre for Drugs and Drug Addiction (EMCDDA) and the European Police Office (EUROPOL), "EMCDDA-Europol 2010 Annual report on the implementation of Council Decision 2005/387/JHA", Lisbon, May 2011.

49-Meltzer, P., Butler, D., Deschamps, J.R. and Madras, B.K., "(4-methylphenyl)-2pyrrolidin-1-yl-pentan-1-one (Pyrovalerone) analogues: a promising class of monoamine uptake inhibitors", Journal of Medicinal Chemistry 49, 2006: 1420-32; other cathinone derivatives, such as amfepramone and bupropion are or have also been used as active pharmaceutical ingredients.

50-Wikström, M., Thelander, G., Nyström, I. and Kronstrand, R, 'Two fatal Intoxications with the New Designer Drug Methedrone (4-Methoxymethcathinone)', Journal of Analytical Toxicology, 2010, 34, 594-98.

51-Collins, M., "Some new psychoactive substances: precursor chemicals and synthesisdriven end-products", Drug Testing and Analysis 3, 2011: 404-16.

52-Bufotenin (a tryptamine closely related to serotonin) was originally found by Wieland in the 1930s; Wieland, H., Konz, W. and Mittash, H., "Die Konstitution von Bufotenin und Bufotenidin. Über Kröten-Giftstoffe VII", Justus Liebigs Annalen der Chemie $513.1,1934$ : 1-25.

53-The structures of psilocin and psilocybin were confirmed by Albert Hoffmann et al. in 1959; Hoffmann, A., Heim. R., Brack, A. and Kobel, H., Experientia 14, 1958: 107-9; Hoffmann, A., et.al., "Psilocybin und Psilocin, zwei psychotrope Wirkstoffe aus mexikanischen Rauschpilzen”, Helvetica Chimica Acta 42, 1959: 1557-72.

54-Alatrash, G., Majhail, N.S. and Pile, J.C., "Rhabdomyolysis after ingestion of "Foxy," a hallucinogenic tryptamine derivative", Mayo Clinic Proceedings 81.4 , 2006: 550-1.

55-Einosuke, T., et.al., "A fatal poisoning with 5-methoxy-N, N-diisopropyltryptamine, Foxy", Forensic Science International 163 , 2006: 152-4; Sklerov, J., et.al., "A fatal intoxication following the ingestion of 5-methoxy-N,N-dimethyltryptamine in an ayahuasca preparation", Journal of Analytical Toxicology 29.8 , 2005: 838-41.

56-Bjoern Moosmann, Volker Auwärter, Designer Benzodiazepines: Another Class of New Psychoactive Substances, Chapter in Handbook of experimental pharmacology 252, October 2018, p. 383, DOI: 10.1007/164_2018_154.

57-Logan BK, Mohr ALA, Friscia M et al., 2017, Reports of adverse events associated with use of novel psychoactive substances, 2013-2016: a review. Anal Toxicol 41(7):573-610. https://doi.org/10.1093/jat/bkx03. 


\section{Abstract \\ New Psychoactive Substances (NPS)}

Nadia Jamal Eldin Zaki

Recent years have seen a steady growth in the availability and consumption of novel psychoactive substances (NPS) across the world. NPS are a range of drugs that have been designed to mimic established illicit drugs, such as cannabis, cocaine, ecstasy and LSD. They are sold as legal, cheap and non-detectable substitutes for controlled drugs, and commonly known by terms such as "designer drugs," "legal highs," or "herbal highs." These drugs are gaining popularity among youth in recent times as they are less expensive and easily available through head shops and internet. A large number of NPS are entering the user market due to the infinite possibility of altering the chemical structures of existing NPS to derive new substances, making them difficult to monitor.

Since 2009, tens of new compounds belonging to six major groups of NPS are introduced into the market each year. The number of compounds belonging to the two most popular groups, synthetic cannabinoids and synthetic cathinones, makes up to two-thirds of the total number of NPS. Different classes of NPS exert their actions by various pharmacological mechanisms, such as the enhancement of central neurotransmission or activation of certain receptors. Activation of NPS can produce a wide spectrum of lifeendangering side effects. NPS may impair cognitive functions, exacerbate or trigger mental disorders and cause severe neurological and cardiovascular symptoms. Definition of new psychoactive substances, different classes of NPS, mode of action, risks and health hazards, the legal situation of NPS were discussed in this article.

We currently present characteristics of these new substances and issues regarding their monitoring and regulatory measures already taken for their control.

These issues would be important for clinicians, law enforcement authorities, and policy makers to consider for mounting an effective response to control the use of NPS. 